DAMTP-2009-37

\title{
Selecting a Model of Supersymmetry Breaking Mediation
}

\author{
S.S. AbdusSalam ${ }^{a}$, B.C. Allanach ${ }^{a}$, M.J. Dolan ${ }^{a}$, F. Feroz ${ }^{b}$ and M.P. Hobson ${ }^{b}$ \\ ${ }^{a}$ DAMTP, CMS, University of Cambridge, Wilberforce Road, \\ Cambridge CBз 0WA, United Kingdom \\ ${ }^{b}$ Cavendish Laboratory, University of Cambridge, JJ Thomson Avenue, \\ Cambridge, CB3 OHE, United Kingdom
}

\begin{abstract}
We study the problem of selecting between different mechanisms of supersymmetry breaking in the Minimal Supersymmetric Standard Model using current data. We evaluate the Bayesian evidence of four supersymmetry breaking scenarios: mSUGRA, mGMSB, mAMSB and moduli mediation. The results show a strong dependence on the dark matter assumption. Using the inferred cosmological relic density as an upper bound, minimal anomaly mediation is at least moderately favoured over the CMSSM. Our fits also indicate that evidence for a positive sign of the $\mu$ parameter is moderate at best. We present constraints on the anomaly and gauge mediated parameter spaces and some previously unexplored aspects of the dark matter phenomenology of the moduli mediation scenario. We use sparticle searches, indirect observables and dark matter observables in the global fit and quantify robustness with respect to prior choice. We quantify how much information is contained within each constraint.
\end{abstract}




\section{Contents}

1 Introduction

1.1 Bayesian inference ....................... 4

2 The Analysis $\quad 6$

2.1 Choice of Prior Probability Distributions . . . . . . . . . . . . 6

2.2 Parameters and ranges . . . . . . . . . . . . . . . . . 7

2.3 The likelihood . . . . . . . . . . . . . . . . . . 7

3 Model Selection $\quad 11$

3.1 A preference for mAMSB, mGMSB, the CMSSM or LVS? . . . . . . . . 12

3.2 Selection of $\operatorname{sign}(\mu) \ldots \ldots \ldots \ldots$

4 mGMSB Parameter Constraints 15

5 mAMSB Parameter Constraints $\quad 17$

6 Constraining Power and Statistical Pull of Observables 20

6.1 Information Content and Constraining Power . . . . . . . . . . . . 20

6.2 Statistical Pull of Observables . . . . . . . . . . . . . . . . 22

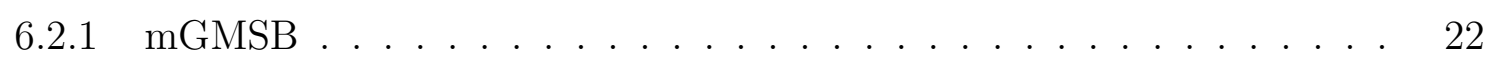

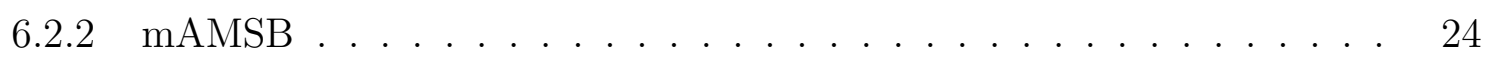

6.3 Combined $\delta a_{\mu}-B R\left(B \rightarrow X_{s} \gamma\right)$ constraint . . . . . . . . . . 26

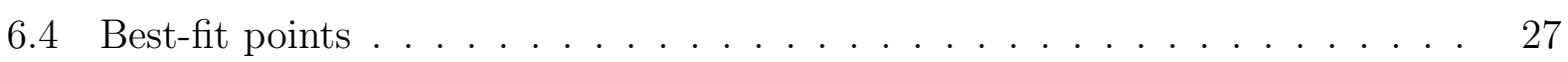

\begin{tabular}{llr}
7 & Conclusion & 29 \\
\hline
\end{tabular}

A Dark Matter Direct Detection $\quad 30$

\section{Introduction}

The Minimal Supersymmetric Standard Model (MSSM) [1, 2] with R-parity can solve the hierarchy problem and provide a candidate dark matter (DM) particle. The absence of observed supersymmetric (SUSY) particles in collider experiments to date implies that if nature is ever supersymmetric then it must be broken. The source of SUSY breaking is a 
priori unknown in a bottom-up approach to particle physics phenomenology. As spontaneous SUSY breaking in the MSSM is not viable [2, one is led to consider SUSY breaking in a hidden sector of the theory communicated via a messenger sector to the MSSM. The choice of messenger sector leaves an imprint on the pattern of SUSY breaking, and therefore on the expected SUSY phenomenology. There are currently many viable soft-SUSY breaking schemes with some common mediation mechanisms including gauge, gravity, anomaly and moduli mediation. There are examples of each class of model which only have a few parameters and can be somewhat constrained by current cosmological and indirect collider data. Sparticles appear in loop contributions to electroweak and $B$-physics observables, affecting their values indirectly. In this paper, we ask the question: is there sufficient power in such combined data to favour one simple model over the other? Such model selection is likely to become even more interesting and important if signals compatible with SUSY are found in the Large Hadron Collider (LHC) experiments.

To date, global Bayesian fits have been performed to the constrained MSSM (CMSSM) [3, 4, 5, 6, 7, 8, 9, 10, 11, 12, large volume string compactification models (LVS) [13], the phenomenological MSSM (pMSSM) [14] and the Non-Universal Higgs model [15]. In any Bayesian fit it is essential to check for robustness by choosing widely different but reasonable prior distributions of the model parameters. For models with more parameters the prior dependence becomes greater, but more precise and direct data will reduce such dependence. The pMSSM has twenty extra-Standard Model (SM) parameters and displays a large priordependence in the fit result:1. Even the CMSSM, with only four extra-SM parameters, shows significant prior dependence and so many aspects of the fits are not robust. The parameterspace fits of the CMSSM and the pMSSM could only be robust with strong enough direct and precise data. The LVS fit, which has two extra-SM parameters, shows approximate prior independence [13]. Here, we shall perform fits to two additional models: minimal anomaly mediated SUSY breaking (mAMSB) and minimal gauge mediated SUSY breaking (mGMSB), each of which have the intermediate number of three parameters. Thus, we ask if the current available data are powerful enough to robustly constrain these models with three parameters additional to the SM as well as ask if it is powerful enough to disfavour any of the models over the others. The MSSM also contains a $\mu$ parameter in the Higgs superpotential, whose magnitude is constrained by the $Z$ boson mass but whose sign is unknown. In the past, MSSM Bayesian model selection has only focused on the extent to which $\mu>0$ is favoured over $\mu<0$ [6, 13, 14, 16]. Thus, our work extends the use of Bayesian model selection to cover different SUSY breaking scenarios.

Aside from the Bayesian fits, there have also been some global profile likelihood (equivalent to minimizing $\chi^{2}$ ) analyses of the CMSSM [7, 10, 17, 18]. Prior dependence does not appear in frequentist interpretations of fits and so one cannot be sure whether they are robust. The Bayesian analyses indicate that such fits are not yet robust with current data. A recent $\chi^{2}$ analysis [19] compared the CMSSM, mGMSB and mAMSB using electroweak and $B$-physics observables while omitting the dark matter constraint. The analysis found that mAMSB was slightly preferred over the two other models by $\Delta \chi^{2}=2$ and that light

\footnotetext{
${ }^{1}$ When we discuss the number of parameters additional to the SM in a model, we refer explicitly to continuous parameters only.
} 
SUSY was slightly favoured by each model. We extend this work in several ways, the most important being that we include dark matter as a constraint. We shall show that the dark matter constraint contains more information content than the other observables. By performing a Bayesian fit with several priors we are able to check robustness of the fit, and, unlike Ref. [19], we incorporate the uncertainties of important SM parameters into our fit.

The parameters of the CMSSM are a flavour blind SUSY breaking scalar mass $m_{0}$, a common gaugino mass $M_{1 / 2}$, a flavour blind SUSY breaking scalar trilinear coupling $A_{0}$ and $\tan \beta$, the ratio of the MSSM Higgs vacuum expectation values (VEVs). Below a grand unification theory (GUT) scale of $M_{G U T} \sim 2 \times 10^{16} \mathrm{GeV}$, the SUSY breaking terms of different flavours evolve separately to the weak scale. In anomaly mediated SUSY breaking (AMSB) [20] SUSY-breaking is communicated to the visible sector via the super-Weyl anomaly. In its original manifestation, pure anomaly mediation suffers from negative slepton mass squared parameters, signalling a scalar potential minimum inconsistent with a massless photon. Minimal AMSB (mAMSB) assumes the existence of an additional contribution to scalar masses $m_{0}$ at $M_{G U T}$ giving it a total of three parameters: the VEV of the auxiliary field in the supergravity multiplet representing the overall sparticles mass scale, $m_{\text {aux }}, m_{0}$ and $\tan \beta$. As advertised above, minimal gauge mediated SUSY breaking (mGMSB) [21] also has three continuous parameters: the overall messenger mass scale, $M_{\text {mess }}$, a visible sector soft SUSYbreaking mass scale, $\Lambda$ and $\tan \beta$. It also contains an additional discrete parameter, namely $N_{\text {mess }}$, the number of SU(5) $5 \oplus \overline{5}$ representations of mediating fields. The example of a moduli mediated model which we consider is the Large Volume Scenario (LVS) derived in the context of IIB flux compactification [22, 23, 24, 25, 13], whose two extra-SM parameters are an overall SUSY breaking mass scale $M_{3 / 2}$ and $\tan \beta$.

The CMSSM is the phenomenologically most studied model in the literature, and so it is useful as a benchmark as to how well other models fare in the fits. It is not clear without further model building how the GUT-scale flavour blind structure of the CMSSM emerges, however. The main motivation for mAMSB and mGMSB is that the flavour problem is solved, since gauge interactions in mGMSB necessarily lead to flavour blind soft terms [21] at the messenger scale, and the mAMSB soft-terms are dominated by flavour diagonal pieces proportional to the gauge couplings [26]. The main motivation for LVS is that it results from a string compactification scenario which has moduli stabilization.

The rest of the paper proceeds as follows: in Section 2 we show the technical aspects of our analysis, including a discussion of model parameters, prior probability distribution functions (PDFs), observables and the calculation of the likelihood. Section 3 presents an analysis of SUSY breaking model selection including the Bayesian evidence results on the preference for the sign of $\mu$, the model selection and the dependence of our results on the dark matter constraint. Sections 4 and 5 detail the phenomenologically viable regions of the parameter spaces of mGMSB and mAMSB, while Section [ discusses the effects of individual observables and presents the best-fit points obtained, concluding with a discussion of the interplay between the anomalous magnetic moment of the muon and the rare branching ratio $B R\left(B \rightarrow X_{s} \gamma\right)$ in the different models. Finally, we discuss the implications of our fits for the direct detection of dark matter in Appendix $\mathrm{A}$. 


\section{$1.1 \quad$ Bayesian inference}

Problems in data analysis generally divide into two categories: parameter estimation and model selection. In parameter estimation problems one is interested in making inferences about the parameters of a given model using the available data and any other prior information. Model selection problems are concerned with distinguishing between different theories describing a given phenomenon. For instance, in the case of the CMSSM, one would like to know whether there is sufficient evidence in the data to rule out the $\mu<0$ branch. Bayesian inference provides a consistent approach to model selection as well as to the estimation of a set of parameters $m$ in a model (or hypothesis) $H$ for the data $d$. It can also be shown that Bayesian inference is the unique consistent generalisation of the Boolean algebra [27].

Assuming some model hypothesis $H$, Bayesian statistics helps update some PDF $p(\underline{m} \mid H)$ of model parameters $\underline{m}$ with data. The prior encodes our knowledge or prejudices about the parameters. Since $p(\underline{m} \mid H)$ is a PDF in $\underline{m}, \int p(\underline{m} \mid H) d \underline{m}=1$, which defines a normalization of the prior. One talks of priors being 'flat' in some parameters, but care must be taken to refer to the measure of such parameters. A prior that is flat between some ranges in a parameter $m_{1}$ will not be flat in a parameter $x \equiv \log m_{1}$, for example. The impact of the data is encoded in the likelihood, or the PDF of obtaining data set $\underline{d}$ from model point $\underline{m}$ : $p(\underline{d} \mid \underline{m}, H) \equiv \mathcal{L}(\underline{m})$. The likelihood is a function of $\chi^{2}$, i.e. a statistical measure of how well the data are fit by the model point. The desired quantity is the PDF of the model parameters $m$ given some observed data $\underline{d}$ assuming hypothesis $H: p(\underline{m} \mid \underline{d}, H)$. Bayes' theorem states that

$$
p(\underline{m} \mid \underline{d}, H)=\frac{p(\underline{d} \mid \underline{m}, H) p(\underline{m} \mid H)}{p(\underline{d} \mid H)},
$$

where $p(\underline{d} \mid H) \equiv \mathcal{Z}$ is the Bayesian evidence, the probability density of observing data set $d$ integrated over all model parameter space. The Bayesian evidence is given by:

$$
\mathcal{Z}=\int \mathcal{L}(\underline{m}) p(\underline{m} \mid H) d \underline{m}
$$

where the integral is over $N$ dimensions of the parameter space $\underline{m}$. Since the Bayesian evidence is independent of the model parameter values $\underline{m}$, it is usually ignored in parameter estimation problems and posterior inferences are obtained by exploring the unnormalized posterior using standard Markov Chain Monte Carlo sampling methods.

In order to select between two models $H_{0}$ and $H_{1}$ one needs to compare their respective posterior probabilities given the observed data set $\underline{d}$, as follows:

$$
\frac{p\left(H_{1} \mid \underline{d}\right)}{p\left(H_{0} \mid \underline{d}\right)}=\frac{p\left(\underline{d} \mid H_{1}\right) p\left(H_{1}\right)}{p\left(\underline{d} \mid H_{0}\right) p\left(H_{0}\right)}=\frac{\mathcal{Z}_{1}}{\mathcal{Z}_{0}} \frac{p\left(H_{1}\right)}{p\left(H_{0}\right)},
$$

where $p\left(H_{1}\right) / p\left(H_{0}\right)$ is the prior probability ratio for the two models, which can often be set to unity but occasionally requires further consideration (see e.g. [28, 29] for cases where the prior probability ratios should not be set to unity). It can be seen from Eq. 3 that Bayesian model selection revolves around the evaluation of the Bayesian evidence. As the average of likelihood over the prior, the evidence automatically implements Occam's razor. A theory 


\begin{tabular}{|c|c|c|c|}
\hline$|\Delta \log \mathcal{Z}|$ & Odds & Probability & Remark \\
\hline \hline$<1.0$ & $\lesssim 3: 1$ & $<0.750$ & Inconclusive \\
1.0 & $\sim 3: 1$ & 0.750 & Weak Evidence \\
2.5 & $\sim 12: 1$ & 0.923 & Moderate Evidence \\
5.0 & $\sim 150: 1$ & 0.993 & Strong Evidence \\
\hline
\end{tabular}

Table 1: The scale we use for the interpretation of model probabilities. Here the 'log' represents the natural logarithm.

with less parameters has a higher prior density since it integrates to 1 over the whole space. Thus, there is an a priori preference for less parameters, unless the data strongly require there be more.

Unfortunately, evaluation of Bayesian evidence involves the multi-dimensional integral in Eq. 2 and thus presents a challenging numerical task. Standard techniques like thermodynamic integration [30] are extremely computationally expensive which makes evidence evaluation typically at least an order of magnitude more costly than parameter estimation. Some fast approximate methods have been used for evidence evaluation, such as treating the posterior as a multi-variate Gaussian centered at its peak (see e.g. [31]), but this approximation is clearly a poor one for highly non-Gaussian and multi-modal posteriors, such as those we sample here. Bridge sampling [32, 33, 34] allows the evaluation of the ratio of Bayesian evidence of two models, but can yield rather imprecise results. The problem can however be tackled by bank sampling [35], but it is not yet clear how precisely bank sampling can calculate the evidence ratio. Various alternative information criteria for model selection are discussed by [36], but the evidence remains our preferred method.

The nested sampling approach, introduced in [37], is a Monte Carlo method targeted at the efficient calculation of the evidence, but also produces posterior inferences as a byproduct. [38, 39] built on this nested sampling framework and introduced the MuLTiNeST algorithm which is efficient in sampling from multi-modal posteriors that exhibit curving degeneracies. MultiNest produces posterior samples and calculates the evidence and its uncertainty. This technique has greatly reduced the computational cost of model selection and the exploration of highly degenerate multi-modal posterior distributions. We employ this technique in this paper.

The natural logarithm of the ratio of posterior model probabilities provides a useful guide to what constitutes a significant difference between two models:

$$
\Delta \log \mathcal{Z}=\log \left[\frac{p\left(H_{1} \mid \underline{d}\right)}{p\left(H_{0} \mid \underline{d}\right)}\right]=\log \left[\frac{\mathcal{Z}_{1}}{\mathcal{Z}_{0}} \frac{p\left(H_{1}\right)}{p\left(H_{0}\right)}\right] .
$$

We summarize the convention we use in this paper in Table 1. 


\section{The Analysis}

\subsection{Choice of Prior Probability Distributions}

While for parameter estimation, the priors become irrelevant once the data are powerful enough, for model selection the dependence on priors always remains (although with more informative data the degree of dependence on the priors is expected to decrease, see e.g. [40]); indeed this explicit dependence on priors is one of the most attractive features of Bayesian model selection. Priors should ideally represent one's state of knowledge before obtaining the data. Rather than seeking a unique 'right' prior, one should check the robustness of conclusions under reasonable variation of the priors. Such a sensitivity analysis is required to ensure that the resulting model comparison is not overly dependent on a particular choice of prior and the associated metric in parameter space, which controls the value of the integral involved in the computation of the Bayesian evidence (for some relevant cautionary notes on the subject see [41]).

We have considered three different prior probability density functions (PDFs) in this analysis. The first is the standard "linear prior" where

$$
p\left(m_{1}\right)=p\left(m_{2}\right)
$$

for $m_{1,2}$ two different points in the parameter space of one of the models under consideration. In particular, the linear priors are flat in the ratio of the two MSSM Higgs vacuum expectation values $(\mathrm{VEVs}), \tan \beta$. It is important to realize that a prior which is flat in one set of parameters $m$ is not necessarily flat in a different set of parameters $m^{\prime}$, say. The two priors will be related by a Jacobian factor such that

$$
p(m)=\left|\frac{d \underline{m}^{\prime}}{d \underline{m}}\right| p\left(m^{\prime}\right) .
$$

One may consider a more fundamental set of parameters to be those involving the quantities that actually appear in the Lagrangian before spontaneous symmetry breaking. Such a set would be flat in the Higgs parameters $B$ and $\mu$, but not in $\tan \beta$. "Natural priors" [7] are priors which are flat in $B$ and $\mu$. The relationship between the two sets of parameters is given by the conditions of electroweak symmetry breaking

$$
\mu B=\frac{\sin 2 \beta}{2}\left(\bar{m}_{H_{1}}^{2}+\bar{m}_{H_{2}}^{2}+2 \mu^{2}\right) \text { and } \mu^{2}=\frac{\bar{m}_{H_{1}}^{2}-\bar{m}_{H_{2}}^{2} \tan ^{2} \beta}{\tan ^{2} \beta-1}-\frac{M_{Z}^{2}}{2},
$$

which lead to the Jacobian factor [7]

$$
J=\frac{M_{Z}}{2}\left|\frac{B}{\mu \tan \beta} \frac{\tan ^{2} \beta-1}{\tan ^{2} \beta+1}\right| .
$$

One may go further to examine the dependence upon the Higgs VEVs, Yukawa couplings and renormalisation group effects in more sophisticated priors [42. We instead focus on a few reasonable but sufficiently different priors in order to check the prior independence of any inference we make. 


\begin{tabular}{|c|c|}
\hline CMSSM & mAMSB \\
\hline $50 \mathrm{GeV} \leq m_{0} \leq 4 \mathrm{TeV}$ & $50 \mathrm{GeV} \leq m_{0} \leq 4 \mathrm{TeV}$ \\
$50 \mathrm{GeV} \leq m_{1 / 2} \leq 2 \mathrm{TeV}$ & $20 \mathrm{TeV} \leq m_{3 / 2} \leq 200 \mathrm{TeV}$ \\
$-4 \mathrm{TeV} \leq A_{0} \leq 4 \mathrm{TeV}$ & \\
\hline \hline mGMSB & LVS \\
\hline $10^{4} \mathrm{GeV} \leq \Lambda \leq 10^{5} \mathrm{GeV}$ & $50 \mathrm{GeV} \leq m_{0} \leq 2 \mathrm{TeV}$ \\
$1.01 \leq M_{\text {mess }} / \Lambda \leq 2 \times 10^{5}$ & \\
\hline
\end{tabular}

Table 2: Ranges for the parameters in mGMSB and the Large Volume Scenario. In mGMSB we also vary the discrete parameter $N_{\text {mess }}$ between 1 and 8 . For all models, $2 \leq \tan \beta \leq 62$.

It will also be the case in this analysis that we wish to estimate the scale of a parameter, rather than its exact value. This is the case for the messenger scale $M_{\text {mess }}$ in gauge mediation, for example. In this case the appropriate prior to use is flat in $\log \left(m_{1}\right)$, and the relevant Jacobian factor is proportional to $m_{1}^{-1}$. We call this the "Jeffreys prior" or simply the "log prior".

\subsection{Parameters and ranges}

Before proceeding we specify the parameter ranges over which we sample for the different models. Firstly, we consider both signs of $\mu$ in our analysis for all models. The ranges over which we vary the continuous model parameters are shown in Table 2. $\tan \beta$ is bounded from below by 2 , values lower than this are in contravention of LEP2 Higgs searches, and from above by 62 , since such large values lead to non-perturbative Yukawa couplings below the GUT scale and calculability is lost. In mGMSB the discrete parameter $N_{\text {mess }}$, the number of messenger multiplets, is varied between 1 and 8 . Higher values of $N_{\text {mess }}$ lead to problems with perturbativity of gauge interactions at the GUT scale [21]. In the CMSSM the unification scale is the standard GUT scale $m_{G U T} \approx 2 \times 10^{16} \mathrm{GeV}$, while for the LVS the soft terms are defined at the intermediate string scale $m_{s} \approx 10^{11} \mathrm{GeV}$ as in [13].

\subsection{The likelihood}

The calculation of the likelihood follows [7] with updated data and additional observables. The constraints we use are all shown in Table 3 along with their respective experimental and theoretical sources. We treat the measurements $D_{i}$ of the observables as independent and we have been careful to eliminate possible correlations between them. We also assume Gaussian errors on all measurements except where explicitly mentioned. The log likelihood of a prediction $p_{i}$ of an observable $i$ is given by

$$
\log \mathcal{L}_{i}=-\frac{\chi_{i}^{2}}{2}-\frac{1}{2} \log (2 \pi)-\log \left(\sigma_{i}\right)
$$




\begin{tabular}{|c|c|c|c|}
\hline Observable & Constraint & Theory & Experiment \\
\hline$\overline{m_{W}[\mathrm{GeV}]}$ & $80.399 \pm 0.027$ & [43, 44] & [45] \\
\hline $\sin ^{2} \theta_{e f f}^{l}$ & $0.23149 \pm 0.000173$ & 43,44 & [46] \\
\hline$\delta a_{\mu} \times 10^{10}$ & $29.5 \pm 8.8$ & {$[47,48,449,50,51,52,53,54$} & 55 \\
\hline$\Omega_{\mathrm{DM}} h^{2}$ & $0.1143 \pm 0.02$ & [47, 48, 49, 50] & 56 \\
\hline$m_{h}[\mathrm{GeV}]$ & $>114.4 \mathrm{GeV}$ & [57] & 58 \\
\hline$\overline{\overline{\Gamma_{Z}^{t o t}}[\mathrm{GeV}]}$ & $2.4952 \pm 0.0023$ & [43] & {$[59,60]$} \\
\hline$R_{l}^{0}$ & $20.767 \pm 0.025$ & [43] & {$[59,60$} \\
\hline$R_{b}^{0}$ & $0.21629 \pm 0.00066$ & [43] & {$[59,60]$} \\
\hline$R_{c}^{0}$ & $0.1721 \pm 0.0030$ & [43] & 59,60 \\
\hline$A_{f b}^{0, b}$ & $0.0992 \pm 0.0016$ & [43] & [59, 60 \\
\hline$\overline{A_{f b}^{0, c}}$ & $0.0707 \pm 0.035$ & [43] & [59, 60] \\
\hline$A_{L R}^{0}(S L D)$ & $0.1513 \pm 0.0021$ & [43] & [59, 60] \\
\hline $\mathcal{A}_{b}$ & $0.923 \pm 0.020$ & 433] & [59, 60 \\
\hline $\mathcal{A}_{c}$ & $0.670 \pm 0.027$ & 43 & 59, 60] \\
\hline 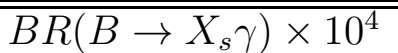 & $3.52 \pm 0.39$ &  & 63 \\
\hline$B R\left(B_{s} \rightarrow \mu^{+} \mu^{-}\right)$ & $<5.8 \times 10^{-8}$ & 477, 48, 49, 50, 64] & 65 \\
\hline$B R(B \rightarrow D \tau \nu)$ & $0.416 \pm 0.138$ & 61,62 & [66] \\
\hline$R_{\Delta M_{s}}$ & $0.85 \pm 0.11$ & 67,68 & 69,63 \\
\hline$R_{B \tau \nu}$ & $1.28 \pm 0.40$ & $61,62,67]$ & 63] \\
\hline$\Delta_{0-}$ & $0.031_{-0.025}^{+0.03}$ & 61,62 & $55,70,71$ \\
\hline$R_{l 23}$ & $1.004 \pm 0.007$ & 61, 62 & 72 \\
\hline$\overline{m_{t}[\mathrm{GeV}]}$ & $172.4 \pm 1.2$ & - & [73] \\
\hline$m_{b}\left(m_{b}\right)^{\overline{M S}}[\mathrm{GeV}]$ & $4.20 \pm 0.07$ & - & 55 \\
\hline$m_{Z}[\mathrm{GeV}]$ & $91.1876 \pm 0.0023$ & - & [55, 59, 60] \\
\hline$\overline{\alpha_{s}^{\overline{M S}}}\left(M_{Z}\right)$ & $0.1172 \pm 0.002$ & - & 74 \\
\hline $1 / \alpha^{\overline{M S}}$ & $127.918 \pm 0.018$ & - & 55] \\
\hline
\end{tabular}

Table 3: Experimental constraints, showing the observables, the constraints applied and the source of the theoretical and experimental values and errors. The first section shows general observables, the second electroweak observables, the third section B-physics constraints and the final section contains SM parameter constraints. The 95\% confidence level (CL) direct sparticle search constraints from [55] were also applied where relevant. 


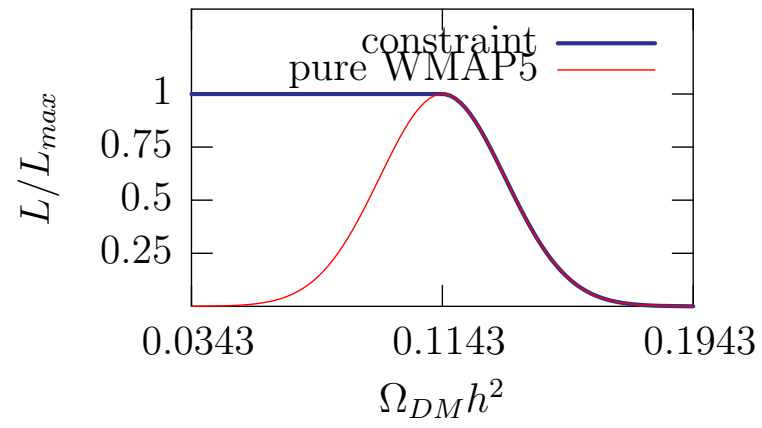

Figure 1: Depiction of our likelihood constraint on the predicted value of $\Omega_{\mathrm{DM}} h^{2}$ due to the lightest neutralino, compared with a simple Gaussian with WMAP5 central value and a $1 \sigma$ uncertainty of 0.02 .

where $\chi_{i}^{2}=\frac{\left(c_{i}-p_{i}\right)^{2}}{\sigma_{i}^{2}}, c_{i}$ is the central experimental value and $\sigma_{i}$ is the standard deviation incorporating both experimental and theoretical uncertainties. All likelihoods except for those of the DM relic density, the Higgs mass and $B R\left(B_{s} \rightarrow \mu^{+} \mu^{-}\right)$are calculated in this way. Details of the constraints applied on the last two exceptions mentioned above can be found in [16]. The $\mathcal{L}_{i}$ considered here are independent, and are multiplied together to calculate the overall likelihood.

For $\Omega_{\mathrm{DM}} h^{2}$, we use two different forms of constraint. Assuming that the DM relic density is composed entirely of the lightest SUSY particle (LSP), one obtains a Gaussian $\mathcal{L}_{\text {DM }}$ with the WMAP5 central value. We call this the 'symmetric $\mathcal{L}_{\mathrm{DM}}$ '. It is of course possible that the neutralino does not make up the entirety of the DM in the universe. For instance, axions or stable moduli could make up the additional component. Indeed, in the Large Volume Scenario, the properties of the lightest Kähler modulus as a DM constituent have been discussed in [75]. In mAMSB, the wino co-annihilation is so efficient that the relic density is generically far below the WMAP value, so that such an extra component is cosmologically necessary. This motivates the use of the 'asymmetric $\mathcal{L}_{\mathrm{DM}}$ ' which is given as:

$$
\mathcal{L}_{\mathrm{DM}}\left(x \equiv \Omega_{\mathrm{DM}} h^{2}\right)= \begin{cases}\frac{1}{c+\sqrt{\pi s^{2} / 2}}, & \text { if } x<c \\ \frac{1}{c+\sqrt{\pi s^{2} / 2}} \exp \left[-\frac{(x-c)^{2}}{2 s^{2}}\right], & \text { if } x \geq c .\end{cases}
$$

where $c$ and $s$ are the mean and $1-\sigma$ error values of the Gaussian $\mathcal{L}_{\text {DM }}$. We take $c=0.1143$ that results from a fit to WMAP5, baryon acoustic oscillations and Type Ia supernovae observations. We also take $s=0.02$ in order to incorporate an estimate of higher order uncertainties in its prediction [76]. For the asymmetric $\mathcal{L}_{\mathrm{DM}}$ we assume that the other dark matter component is R-parity even, so therefore we apply the constraint that the LSP must not be charged or coloured, since then it would be stable and have appeared in rare isotope searches. A diagram of the resulting likelihood penalty is displayed in Fig. 1 .

In mGMSB the gravitino is the LSP. The gravitino mass $m_{3 / 2}$ depends on the scale of SUSY breaking and is usually in the range of a few eV up to a few hundred keV. This 
property has often been cited as an attractive feature of mGMSB theories, as the low mass of the gravitino indicates that gravity mediated effects are much smaller than gauge mediated effects, so that flavour changing neutral currents are naturally suppressed. The gravitino contribution to the relic density is given by [77]

$$
\Omega_{3 / 2} h^{2} \approx \frac{m_{3 / 2}}{\mathrm{keV}}\left[\frac{100}{g_{*}\left(T_{f}\right)}\right]
$$

where $g_{*}\left(T_{f}\right)$ is the number of massless degrees of freedom at the gravitino freeze-out temperature, and for SUSY models is in the range 100-200 [21]. We see from Eq. 10 that achieving the WMAP value of the relic density requires a gravitino at the lower end of the mass range, in particular less than $1 \mathrm{keV}$. Constraints on structure formation and WMAP [78] are now strong enough, however, to rule out the resultant warm DM. Heavier gravitinos which evade this bound would be over abundant compared to observation. Although it may be possible to dilute the gravitino relic density by late entropy production, we wish to keep our analysis as general as possible without confining ourselves to a specific change in the physics of the early universe. Accordingly, in our analysis we do not impose any DM constraint on mGMSB.

The combined log likelihood is the sum of the individual likelihoods,

$$
\log \mathcal{L}^{t o t}=\sum_{i} \log \mathcal{L}_{i}
$$

To calculate the MSSM spectrum we use Softsusy2.0.18 [79] which calculates the spectrum of the CMSSM, mAMSB and mGMSB. By modifying the unification scale from $m_{G U T}$ to $m_{\text {string }} \sim 10^{11} \mathrm{GeV}$ and the gauge coupling boundary conditions Softsusy can also provide the spectrum in the LVS case. Parameter space points which violate the current sparticle exclusion bounds of [55, do not break electroweak symmetry correctly or have tachyonic sparticles are assigned zero likelihood. Points which have a charged LSP are rejected?. If a point survives the cuts above, it is passed via the SUSY Les Houches Accord [81] to microMEGAS2 2 [47, 48, 49, 50], SuperIso2. 3 [61, 62] and SusyPOPE [44]. From micromEGAS we obtain the DM relic density, the rare branching ratio $B R\left(B_{s} \rightarrow \mu^{+} \mu^{-}\right)$, the SUSY component $\delta a_{\mu}$ of the anomalous magnetic moment of the muon $(g-2)_{\mu}$ and DM direct detection rates. To the one-loop value of $a_{\mu}$ calculated by microMEGAS, we add the logarithmic piece of the quantum electro-dynamics 2-loop calculation, the 2-loop stop-Higgs and charginostop/bottom pieces and the recently discovered two-loop effect due to a shift in the muon Yukawa coupling and proportional to $\tan ^{2} \beta$ [51, 52, 53, 54]. From SuperIso2 3 the branching ratios $B R\left(B \rightarrow X_{s} \gamma\right), B R(B \rightarrow D \tau \nu)$, the quantities $R_{\Delta M_{s}}, R_{l 23}, R_{B \tau \nu}$ and the isospin asymmetry $\Delta_{0-}$ are obtained 3 . SusyPOPE provides all of the observables listed in the second part of Table 3 .

\footnotetext{
${ }^{2}$ Due to the small neutralino-chargino splitting in mAMSB we have been careful to reject any points that would violate the long-lived charged stable particle bounds from Tevatron, which requires $\Delta m=$ $m_{\chi_{1}^{+}}-m_{\chi_{1}^{0}}>50 \mathrm{MeV}$. In fact, we find that this bound does not constrain the mAMSB parameter space since mAMSB predicts larger splittings [80.

${ }^{3}$ We note that in the process of preparing this paper and after our fits were performed a new version of SusyBSG 82 appeared. This more accurate calculation could result in a change in our $B R\left(B \rightarrow X_{s} \gamma\right)$ prediction of up to $0.13 \times 10^{-4}$.
} 
We present our data in the form of marginal posteriors in one and two dimensions, where the unseen dimensions have been integrated over. To do this integration numerically we divide the range of the parameter in which we are interested into a series of bins, and then count the number of samples which fall into each bin. We use 60 bins per parameter, which is a trade-off between parameter resolution and unwanted statistical noise from finite sampling effects. We shall also discuss the profile likelihood at various stages. The profile likelihood is often shown in one dimension, and plots the maximum of the likelihoods of the samples that fall in each bin. This statistic is equivalent to plotting minimum $\chi^{2}$ in various directions of parameter space, and shows where the data are fitted best, but does not take into account volume effects of the less best-fit points. Everywhere in this paper, we take best-fit to mean the highest likelihood (or equivalently, lowest $\chi^{2}$ ). MultiNest is optimized for calculating Bayesian evidence values, and also typically gives a reasonable sampling for posterior evaluation. However, the profile likelihood is extracted by MULTiNeST with a lot of noise. It was found by Ref. [10] to depend upon the prior, which it should not do for a large enough sampling. Therefore, here we do not explicitly include plots of the profile likelihood, preferring instead to mention its behaviour at various points in the text.

We have taken care that all the two-sided constraints applied in the likelihood are normalised to 1. For one-sided constraints such as sparticle exclusion limits, the likelihood is zero below the $95 \%$ confidence limit, and 1 above that limit. The likelihoods we apply for $B R\left(B_{s} \rightarrow \mu^{+} \mu^{-}\right)$and $m_{h}$ are more complicated, but they both asymptote to 1 in the limit of zero branching ratio and large Higgs mass respectively. While we focus on the statistically meaningful difference between evidences in our fits, by specifying the normalisation of the likelihood and providing also the absolute values of the evidence in our tables of results we hope to encourage other groups, by following our calculation, to directly compare Bayesian evidences calculated for other models with the results presented herein.

\section{Model Selection}

In this section we discuss whether current data prefer one model over the others in the set mAMSB, mGMSB, the CMSSM and LVS. We also present our results on quantification of the preference of the fits for $\mu>0$. In both these cases, we do the analysis with 'symmetric $\mathcal{L}_{\mathrm{DM}}$ ' as well as 'asymmetric $\mathcal{L}_{\mathrm{DM}}$ ' (see Section 2.3). Strictly speaking, a calculation of the Bayesian evidence with asymmetric dark matter constraints is inherently unfair unless the relic density of the additional DM component is included, with an associated reduction in the evidence due to the Occam's razor effect. We shall here make model selections between models where the asymmetric likelihood penalty has been applied to all of the models in question. Thus, although the Occam's razor effect is neglected, it should affect all of the models being compared in approximately the same way and so the comparison should remain unaffected. We also assume that the prior probabilities of each model are equal and independent of the sign of $\mu$. 


\begin{tabular}{|c|c|c|c|c|c|c|}
\hline & \multicolumn{3}{|c|}{ symmetric $\mathcal{L}_{\text {DM }}$} & \multicolumn{3}{c|}{ asymmetric $\mathcal{L}_{\text {DM }}$} \\
\hline \hline Model/Prior & linear & $\log$ & natural & linear & $\log$ & natural \\
\hline CMSSM & $8.0 \pm 0.1$ & $7.9 \pm 0.1$ & $10.3 \pm 0.1$ & $0.0 \pm 0.1$ & $1.0 \pm 0.1$ & $1.3 \pm 0.1$ \\
mAMSB & $0.4 \pm 0.1$ & $0.6 \pm 0.1$ & $0.0 \pm 0.1$ & $5.1 \pm 0.1$ & $6.0 \pm 0.1$ & $5.0 \pm 0.1$ \\
LVS & $8.7 \pm 0.1$ & $8.9 \pm 0.1$ & $11.8 \pm 0.1$ & $2.9 \pm 0.1$ & $3.0 \pm 0.1$ & $3.1 \pm 0.1$ \\
\hline
\end{tabular}

Table 4: $\log$ evidences $(\Delta \log \mathcal{Z})$ for mAMSB, LVS and the CMSSM for both signs of $\mu$. Symmetric $\mathcal{L}_{\mathrm{DM}}$ labels the assumption that the DM relic density is composed entirely of the LSP and asymmetric $\mathcal{L}_{\text {DM }}$ labels the assumption that the LSP forms only a part of the DM relic density. The $\log$ evidence of the natural prior $\mathrm{mAMSB}, \log Z_{s}=67.3$ and the log evidence of the linear prior CMSSM, 76.7 have been subtracted from all entries in the symmetric $\mathcal{L}_{\mathrm{DM}}$ and asymmetric $\mathcal{L}_{\mathrm{DM}}$ respectively.

\subsection{A preference for mAMSB, mGMSB, the CMSSM or LVS?}

We now discuss to what extent the mAMSB, mGMSB, the CMSSM, and LVS model are preferred over one another by the current data. In this subsection, we marginalise over the sign of $\mu$.

The $\log$ evidence values $(\log \mathcal{Z})$ for mAMSB, LVS and the CMSSM are the most important results of this paper and they are shown in Table 4. mAMSB is strongly favoured over the $\operatorname{CMSSM}(\Delta \log \mathcal{Z}>5)$ and moderately favoured over LVS $(\log \Delta \mathcal{Z}>2)$ for asymmetric $\mathcal{L}_{\mathrm{DM}}$, a result which is approximately prior independent. However, mAMSB is almost decisively ruled out for symmetric $\mathcal{L}_{\mathrm{DM}}(\Delta \log \mathcal{Z}<-7)$. Although mAMSB with a purely thermal relic density is decisively disfavoured we have not taken into account the non-thermal component of the relic density due to decays of the heavy gravitino in mAMSB which for, some values of the inflationary reheating temperature, could saturate the WMAP bounds. As mentioned above, in mAMSB, the degeneracy between the LSP and next-to-LSP (NLSP) chargino causes extremely efficient co-annihilation of the LSP to near zero relic density over the whole parameter space. Thus, the entire parameter space of mAMSB passes the asymmetric $\mathcal{L}_{\mathrm{DM}}$ constraint and it is strongly preferred. On the other hand the LSP relic density in most of the mAMSB parameter space is much lower than the central value inferred by WMAP and hence mAMSB is almost ruled out if one assumes that the DM relic density is composed entirely of the LSP. There is a weak to moderate preference for LVS over the CMSSM depending on the prior and whether the symmetric or asymmetric form of $\mathcal{L}_{\mathrm{DM}}$ is taken.

To quantify the extent to which these results depend on the DM constraint, we calculate the Bayesian evidence ratios for mAMSB, mGMSB, LVS and the CMSSM without this constraint and list them in Table 5. It is clear from these evidence values that the results are completely inconclusive in the absence of the DM constraint and hence it can be concluded that it does indeed dominate our model selection results. Ref. [19] concluded on the basis of a $\chi^{2}$ minimisation with a subset of the observables included in the present paper and no DM constraint that mAMSB was slightly preferred over the other two models. Table 5 indicates that even such a weak inference cannot yet be made due to non-robustness of fits in mAMSB, since the preference for or against it depends upon the form of the prior. 


\begin{tabular}{|c|c|c|c|}
\hline Model/Prior & linear & log & natural \\
\hline \hline CMSSM & $0.9 \pm 0.1$ & $1.0 \pm 0.1$ & $1.1 \pm 0.1$ \\
mAMSB & $1.1 \pm 0.1$ & $1.2 \pm 0.1$ & $2.9 \pm 0.1$ \\
mGMSB & $1.4 \pm 0.1$ & $1.5 \pm 0.1$ & $0.0 \pm 0.1$ \\
LVS & $1.1 \pm 0.1$ & $0.8 \pm 0.1$ & $1.5 \pm 0.1$ \\
\hline
\end{tabular}

Table 5: $\log$ evidences $(\log \mathcal{Z})$ for mAMSB, mGMSB, LVS and the CMSSM for both signs of $\mu$ and without imposing the $\Omega_{\mathrm{DM}} h^{2}$ constraint. A constant value (the log evidence of mGMSB using natural priors, 78.2) has been subtracted from all the log evidence values.

\begin{tabular}{|c|c|c|c|c|c|c|}
\hline & \multicolumn{3}{|c|}{ symmetric $\mathcal{L}_{\text {DM }}$} & \multicolumn{3}{c|}{ asymmetric $\mathcal{L}_{\text {DM }}$} \\
\hline \hline Model/Prior & linear & $\log$ & natural & linear & $\log$ & natural \\
\hline CMSSM & $1.2 \pm 0.1$ & $2.4 \pm 0.1$ & $0.4 \pm 0.1$ & $1.3 \pm 0.1$ & $2.4 \pm 0.1$ & $0.4 \pm 0.1$ \\
mAMSB & $1.4 \pm 0.1$ & $2.5 \pm 0.1$ & $0.4 \pm 0.1$ & $1.9 \pm 0.1$ & $3.4 \pm 0.1$ & $0.6 \pm 0.1$ \\
LVS & $3.2 \pm 0.1$ & $3.1 \pm 0.1$ & $2.6 \pm 0.1$ & $3.6 \pm 0.1$ & $3.9 \pm 0.1$ & $3.3 \pm 0.1$ \\
\hline \hline & \multicolumn{4}{|c|}{} & \multicolumn{3}{c|}{ No DM constraint } \\
\hline mGMSB & \multicolumn{4}{|c|}{$1.7 \pm 0.1$} & $2.2 \pm 0.1$ & $1.4 \pm 0.1$ \\
\hline
\end{tabular}

Table 6: Difference in $\log$ evidences $(\Delta \log \mathcal{Z})$ for $\mu>0$ and $\mu<0$ for the CMSSM, mAMSB and the LVS model. A positive value for a model indicates a preference for $\mu>0$. 'Symmetric $\mathcal{L}_{\mathrm{DM}}$ ' labels the assumption that the DM relic density is composed entirely of the LSP and 'asymmetric $\mathcal{L}_{\mathrm{DM}}$ ' labels the assumption that the LSP forms only a part of the DM relic density.

\subsection{Selection of $\operatorname{sign}(\mu)$}

We summarize the amount of preference for $\mu>0$ in mAMSB, the CMSSM and LVS in Table 6. We list the Bayes factors, $\Delta \log \mathcal{Z}$ in favour of $\mu>0$ for these three models. Using the Jeffreys scale defined in Table 1, it is evident that although there is a positive but not strong evidence in favour of $\mu>0$ for all three models, the extent of the preference depends quite strongly on the priors used. Although there are small numerical differences due to the constraints being updated and different prior ranges taken, the CMSSM $\Delta \log \mathcal{Z}$ values lead to the same conclusions as previous determinations [6, 13, 14, 16]: the preference for $\mu>0$ is moderate at best, and quite prior dependent. This dependence on the prior is a clear sign that the data are not yet of sufficiently high quality to be able to distinguish between these models unambiguously and hence it is not justified to ignore the $\mu<0$ branch in any of the models under consideration, despite the fact that $(g-2)_{\mu}$ favours positive $\mu$ by around $3.4 \sigma$. 

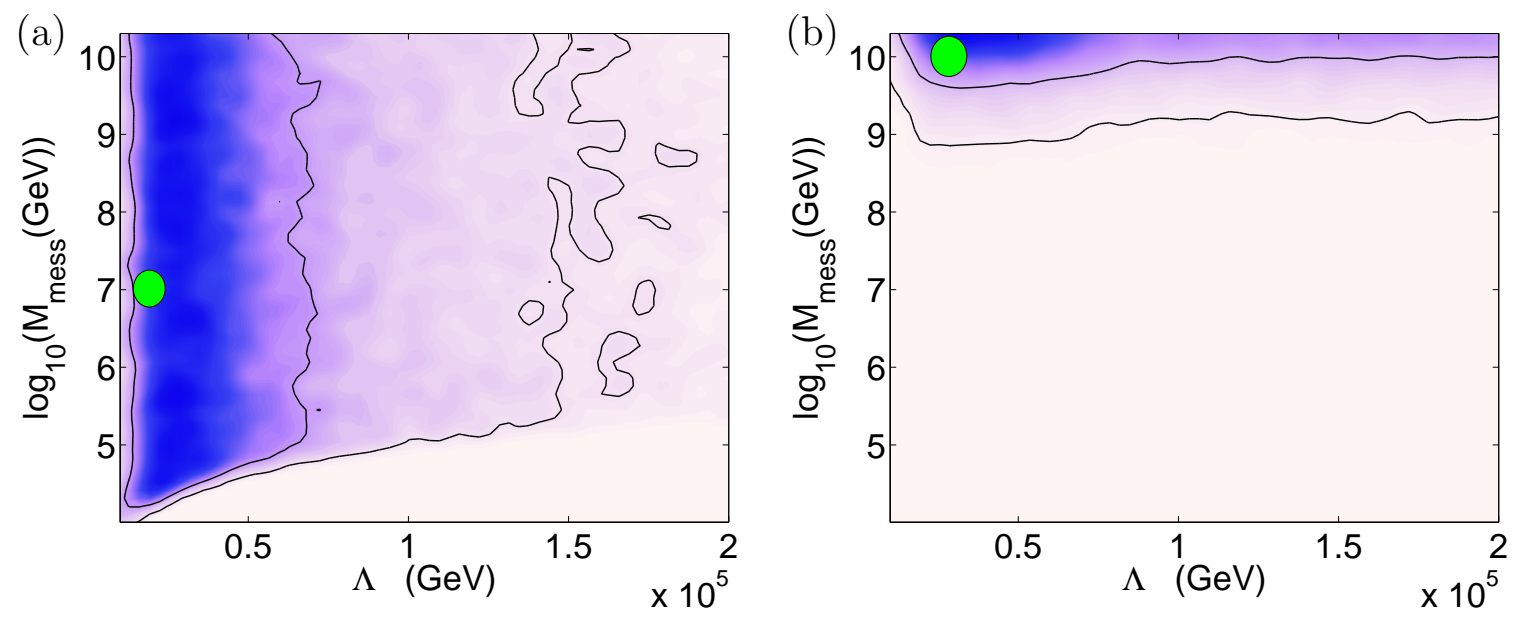

Figure 2: 2D marginalised posterior PDFs for mGMSB in the $\log _{10}\left(M_{\text {mess }}\right)-\Lambda$ plane for (a) $\log$ priors and (b) natural priors with $68 \%$ and $95 \%$ Bayesian credibility intervals. Both plots are marginalized over both signs of $\mu$. Green dots show the position of the best-fit point for each prior choice sample.
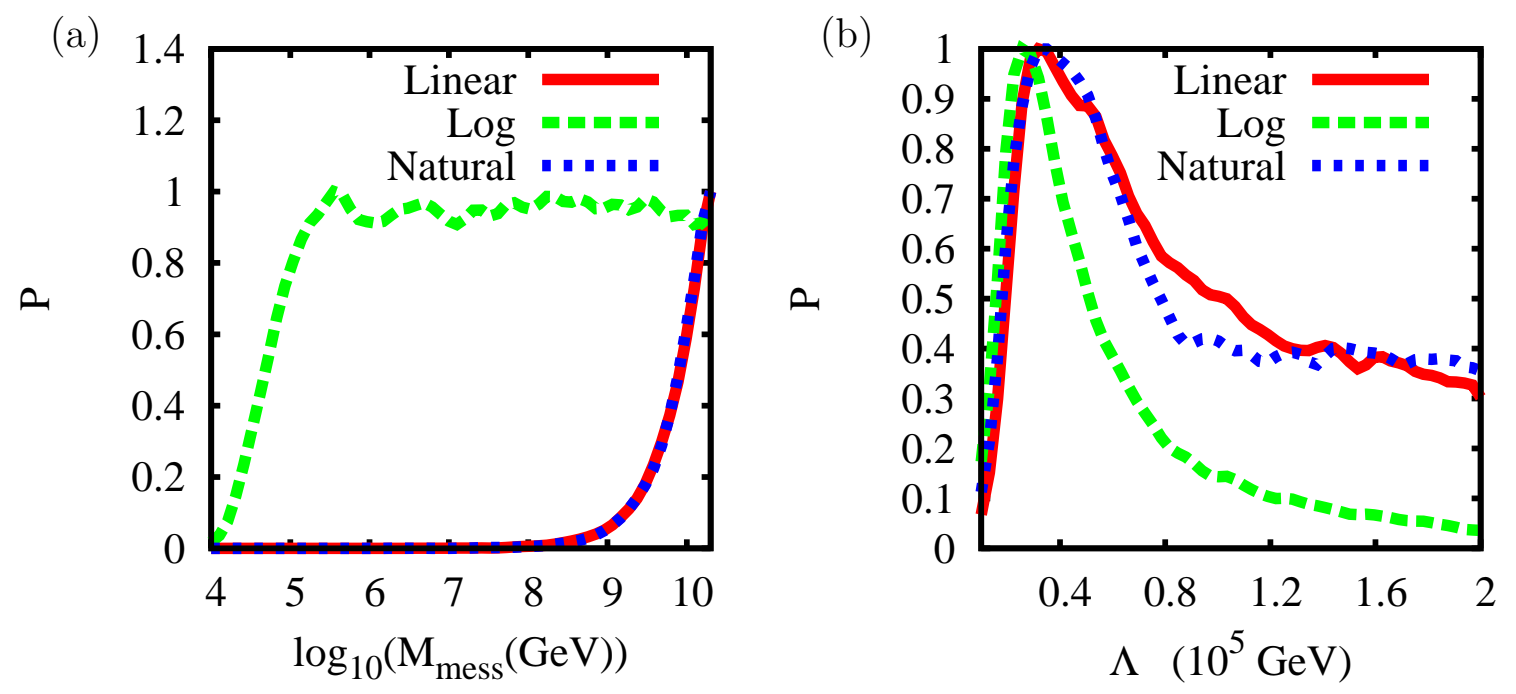

Figure 3: mGMSB 1D marginalized posterior PDFs over (a) $\log _{10}\left(M_{\text {mess }}\right)$ and (b) $\Lambda$ for linear, $\log$ and natural priors. Both plots are marginalized over both signs of $\mu$. Both here and in future one-dimensional marginalizations, the vertical axis has been normalized so that the maximum posterior PDF is one. 


\section{4 mGMSB Parameter Constraints}

We now discuss the constraints on mGMSB coming from the global fit. These have not appeared in the literature before, except for in Ref. [19], where a $\chi^{2}$ minimisation was performed, and so prior dependence was not quantified. Fig. 2 shows the marginalized joint posterior PDFs for mGMSB in the $\log _{10}\left(M_{\text {mess }}\right)-\Lambda$ plane for logarithmic and natural priors. We have also marginalized over the messenger index $N_{\text {mess }}$. The most striking feature of the plots shown is that while the $2 \mathrm{D}$ posterior with the log prior is flat in the logarithm of the messenger scale $M_{\text {mess }}$, for the natural priors there is a very strong peak at the highest values of $M_{\text {mess }}$. This is due to a volume effect. With a linear or natural prior, smaller values of $M_{\text {mess }}$ occupy a smaller prior volume, which pushes the posterior for $M_{\text {mess }}$ down. The $\log$ priors sample more from the region of low $M_{\text {mess }}$, due to the suppression from the log prior measure, and are in rough agreement with the profile likelihood. The excluded region at low values of the messenger scale in Fig. 2(a) is due to the requirement that $M_{\text {mess }}>\Lambda$, since if this is not the case the messengers are tachyonic. In order to understand why $M_{m e s s}$ is only very weakly constrained by the data, consider the spectrum resulting from gauge mediation. To leading-log order, the gaugino masses at the scale $M_{\text {mess }}$ are

$$
M_{\tilde{\lambda}_{i}}\left(M_{m e s s}\right)=k_{i} N_{m e s s} \Lambda \frac{\alpha_{i}\left(M_{m e s s}\right)}{4 \pi} g\left(\Lambda / M_{m e s s}\right),
$$

where $k_{i}=(5 / 3,1,1)$ and $k_{i} \alpha_{i}$ (no summation) are all equal at the GUT scale, and $\alpha_{i}$ are the gauge coupling constants. The messenger scale threshold function $g$ can be found in [21] and $g(x) \rightarrow 1$ when $\Lambda \ll M_{\text {mess }}$. This is the case throughout most of the mGMSB parameter space that we study. In this limit one can also approximate the scalar masses by

$$
m_{\tilde{f}}^{2}\left(M_{m e s s}\right)=2 N_{m e s s} \sum_{i=1}^{3} C_{i} k_{i} \frac{\alpha_{i}^{2}\left(M_{m e s s}\right)}{(4 \pi)^{2}} \Lambda^{2} .
$$

where $C_{i}$ are the quadratic Casimir operators of the relevant gauge groups [21]. The dependence on $M_{\text {mess }}$ is somewhat subtle. Increasing $M_{\text {mess }}$ leads to different initial conditions for $\alpha_{i}\left(M_{\text {mess }}\right)$ and also increases how far the renormalisation group equations (RGEs) must be evolved to reach the low scale. As pointed out in [83] these effects cancel each other out in the gaugino masses since the gaugino masses obey the same one-loop RGEs as the gauge couplings. There is however an effect on the scalar masses, leading to a decrease in the squark masses and an increase in the slepton masses at larger values of $M_{\text {mess }}$. This statement does not apply to the stop masses, which do depend somewhat on $\tan \beta$ due to mixing. Therefore the mGMSB spectrum is fairly independent of $M_{\text {mess }}$. We remind the reader that for the mGMSB fits, no DM constraint is applied.

Fig. 3(b) shows the 1D PDF posterior for the SUSY breaking scale $\Lambda$. All sets of priors have a strong peak at around $3 \times 10^{4} \mathrm{GeV}$ before falling away at higher values of $\Lambda$. Since $\Lambda$ is directly related to the scale of the SUSY spectrum, high values of $\Lambda$ lead to a heavier

spectrum. Such a spectrum fits $(g-2)_{\mu}$ and $B R(b \rightarrow s \gamma)$ worse than a lighter spectrum, as we shall show in section 6.3 .

We next turn to $\tan \beta$, for which we present the posterior PDFs in Fig. 4(a). The posterior PDFs for the logarithmic prior have the same shape as the profile likelihood, with 

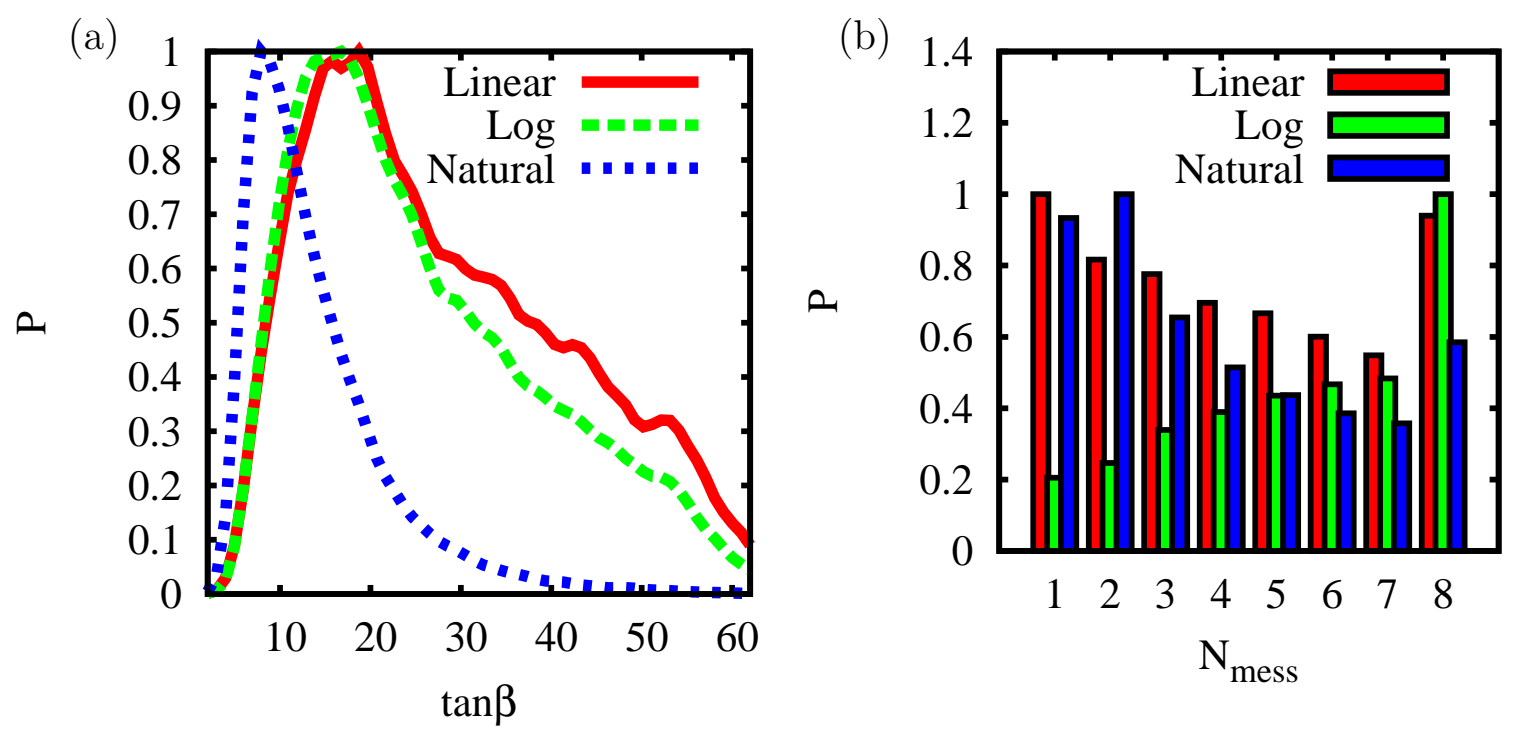

Figure 4: mGMSB 1D marginalized posterior PDFs for (a) $\tan \beta$ and (b) the messenger index $N_{\text {mess }}$ for log, linear and natural priors.

both sharply rising from the allowed value of $\tan \beta=2$ to a most likely value just under 20 and then gradually falling away as $\tan \beta$ increases, a feature common to both branches of $\mu$. It is primarily the anomalous magnetic moment of the muon that is pulling the model towards high $\tan \beta$, where a significant SUSY component can fit the data. SUSY contributions to $(g-2)_{\mu}$ are dominated by one loop diagrams involving chargino-sneutrino or neutralino-smuon in the loop. These are approximately

$$
\left.(g-2)_{\mu}^{S U S Y} \approx \frac{m_{\mu}^{2} \mu \tan \beta}{16 \pi^{2}}\left(g_{1}^{2} F_{1} M_{1}+g_{2}^{2} F_{2} M_{2}\right)\right)
$$

where $m_{\mu}$ is the muon mass and $F_{1,2}$ are positive definite functions of sparticle masses, which scale as $1 / M_{S U S Y}^{4}$ in the limit of heavy degenerate sparticles of mass $M_{S U S Y}$. With natural priors the behaviour is similar, except that the peak occurs at a lower value of $\tan \beta$ driven by the fact that natural priors prefer small $\tan \beta$, which can be seen by inspecting the Jacobian factor in Eq. 7) Fig. 4(b) shows the posterior PDFs for the discrete messenger index $N_{\text {mess }}$. The log priors prefer a large number of messenger multiplets, with the most likely value of $N_{\text {mess }}$ clearly being 8 . Since the log prior prefers light values of the neutralino and chargino masses, a good fit to the anomalous magnetic moment of the muon is also preferred by having relatively light scalars, so that the smuon and sneutrino diagrams may contribute significantly. From Eqs. 12 and 13, a slepton mass divided by a weak gaugino mass is proportional to $1 / \sqrt{N_{\text {mess }}}$ and so high $N_{\text {mess }}$, i.e. light sleptons is preferred. This preference is less strong for the heavier spectra with the linear or natural priors, where it is more difficult to fit $(g-2)_{\mu}$. It also has to compete with volume effects coming from the LEP2 Higgs constraint, which prefers heavy stops and therefore low $N_{\text {mess }}$.

To summarize, all of the mGMSB parameters show significant prior dependence, even when each is marginalized down to one dimension. We decline to present posterior PDFs of sparticle masses, since they show the same non-robustness with respect to changing the priors. 


\section{5 mAMSB Parameter Constraints}

In this section we discuss the effects of the observable constraints on the anomaly mediated SUSY breaking parameter space. All supergravity theories suffer from a Weyl (rescaling) anomaly which leads to soft breaking terms for the visible sector. These contributions are usually rendered negligible by the usual gravity mediated soft breaking terms. If the gravity mediated terms are suppressed, perhaps by a mechanism similar to that originally suggested in the brane model of [20] then the SUSY breaking is dominated by the anomaly mediated terms to leading order. In pure AMSB the slepton masses suffer from being tachyonic. One way this can be ameliorated while avoiding the SUSY flavour problem is by introducing a new universal mass parameter $m_{0}$ at the GUT scale which lifts the slepton masses. We define the GUT scale to be the scale of electroweak gauge unification. The GUT-scale soft breaking terms for the gauginos, scalars and trilinear couplings are then given by

$$
\begin{gathered}
M_{i}=\frac{\beta_{g_{i}}}{g_{i}} m_{3 / 2}, \\
m_{\tilde{Q}}^{2}=-\frac{1}{4}\left(\frac{\partial \gamma}{\partial g_{i}} \beta_{g_{i}}+\frac{\partial \gamma}{\partial y} \beta_{y}\right) m_{3 / 2}^{2}+m_{0}^{2}, \\
A_{y}=-\frac{\beta_{y}}{y} m_{3 / 2},
\end{gathered}
$$

where $\beta_{g_{i}}$ is the beta function of the $i^{\text {th }}$ SM gauge group, $\gamma$ is the anomalous dimension of the respective scalar wave-function and $y$ and $\beta_{y}$ are Yukawa couplings and Yukawa beta functions respectively. Eqs. 15,17 constitute the "minimal AMSB" (mAMSB) SUSY breaking assumption. Its spectrum is specified by three continuous free parameters: the gravitino mass $m_{3 / 2}$, the parameter $m_{0}$ and $\tan \beta$, along with the sign of the $\mu$ parameter. Our mAMSB fits use the 'asymmetric' DM constraint shown in Fig. 1, since the mAMSB neutralino relic density is too low to be the sole component of the dark matter.

Fig. [5(a,c,e) shows the posterior PDFs for linear priors in the $m_{3 / 2}-m_{0}, m_{3 / 2}$-tan $\beta$ and $m_{0}$-tan $\beta$ planes while Fig. [5(b,d,f) shows the same plots for natural priors. The dark matter constraint is asymmetric in both cases. There is clearly a strong prior dependence in all parameters. This is due to the pull of the natural priors to low values of $\tan \beta$ and to high values of $B / \mu$, which occurs near the focus point at high $m_{0}$ [84]. The linear priors identify a different region of high probability at low $m_{0}$ and low $m_{3 / 2}$ leading to a light sparticle spectrum capable of satisfying the constraints from $(g-2)_{\mu}$ and $B R\left(B \rightarrow X_{s} \gamma\right)$. Log prior posteriors are omitted for brevity, but they are similar to the linear prior results, except that the log priors have an even stronger preference for low $m_{0}$ and $m_{3 / 2}$.

There are three significant regions of parameter space which have been ruled out already. The first is the triangular region at low $m_{0}$ and moderate to high values of $m_{3 / 2}$. This is ruled out by the direct search constraints on the sleptons. The large values of $m_{3 / 2}$ and small $m_{0}$ lead to low (or even imaginary) values of the slepton masses in Eq. 16. The disallowed strip at low values of $m_{3 / 2}$ and all values of $m_{0}$ is due to gaugino masses being too low. The region defined by $\tan \beta \gtrsim 50$ is decisively disfavoured by a combination of 

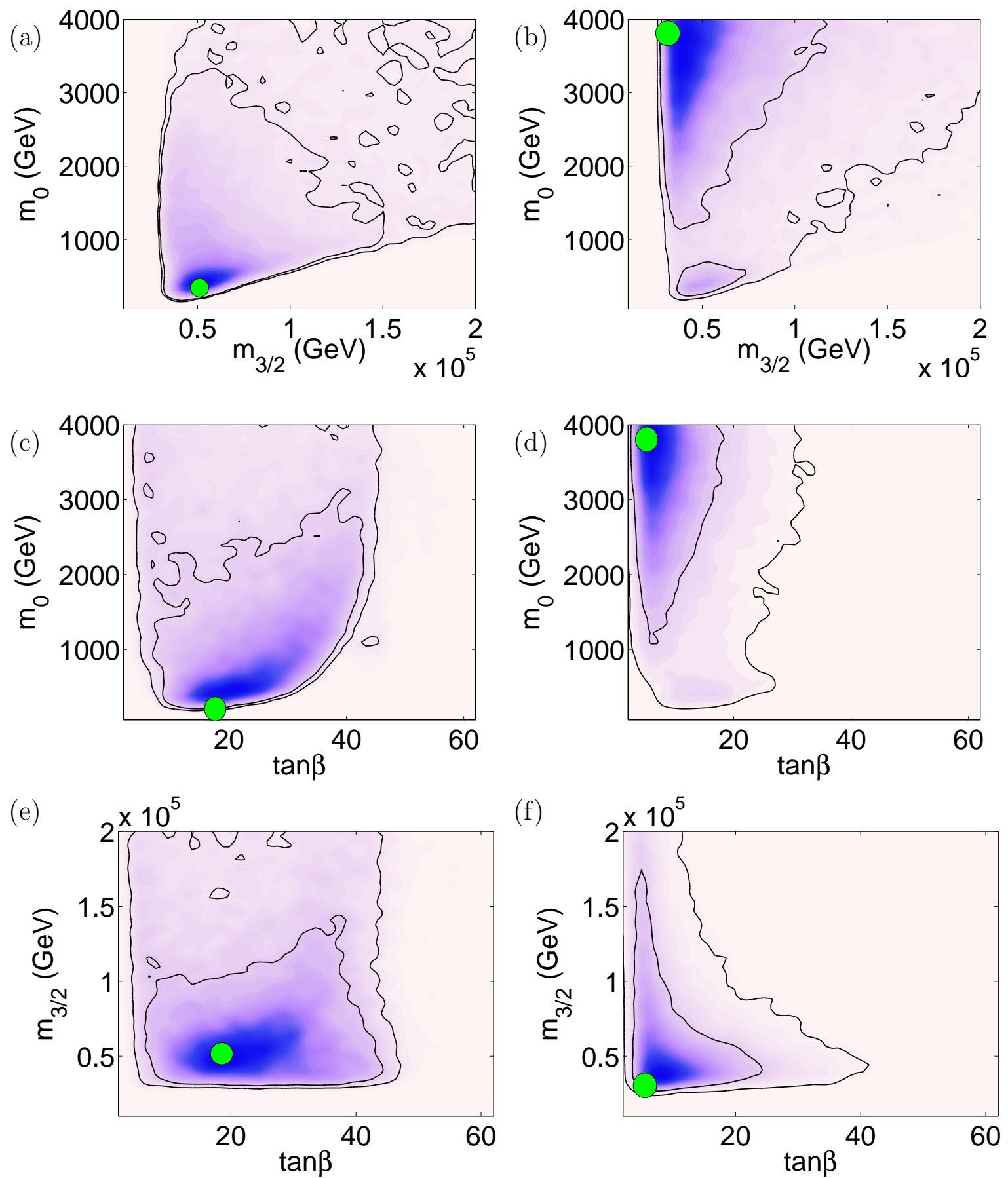

Figure 5: mAMSB marginalised 2D posterior PDFs in the $m_{0^{-}} m_{3 / 2}, m_{0^{-}} \tan \beta$ and $m_{3 / 2^{-}}$ $\tan \beta$ planes for linear priors (left-hand side pictures) and natural priors (right-hand side pictures) with asymmetric $\mathcal{L}_{D M}$. The green dots mark the best-fit points in each prior case sample. $68 \%$ and $95 \%$ Bayesian credibility regions are shown as the inner and outer contours respectively. 

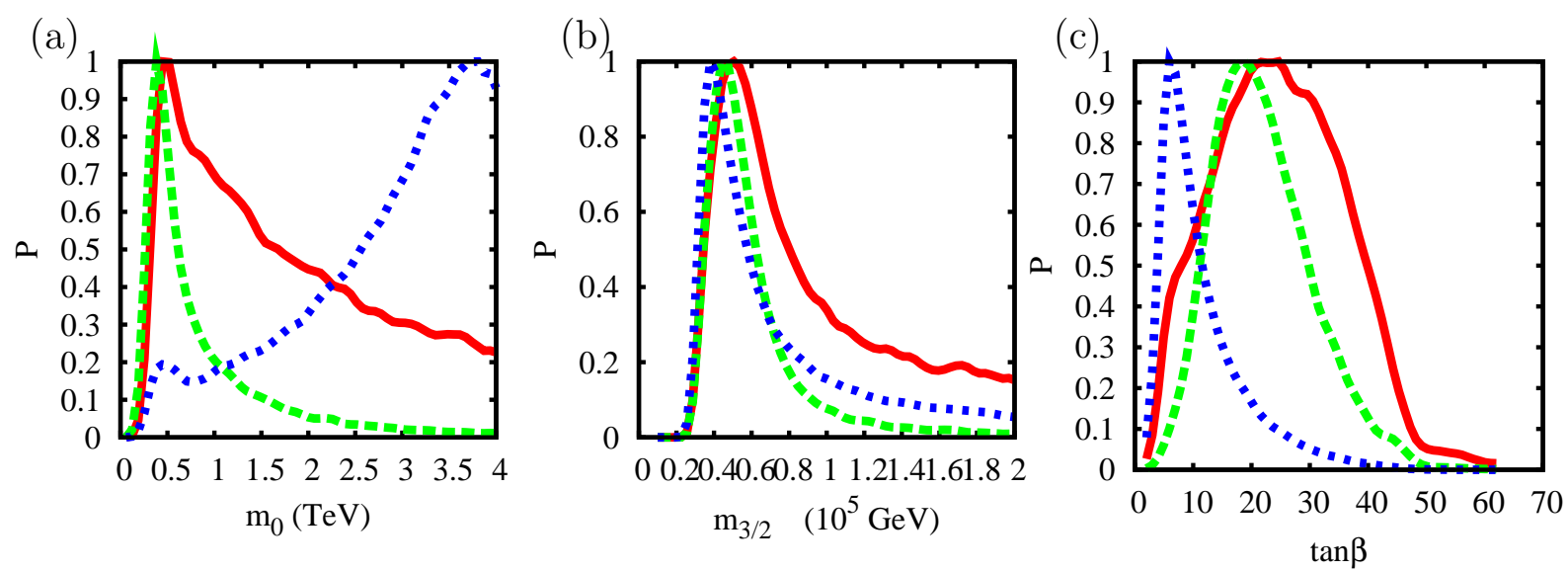

Figure 6: mAMSB marginalized 1D posterior PDFs for (a) $m_{0}$, (b) $m_{3 / 2}$ and (c) $\tan \beta$ for different priors. Red (solid) lines represent linear priors, green (dashed) lines for log priors and blue (dotted) lines for natural priors. All plots are for asymmetric $\mathcal{L}_{D M}$.

related circumstances: tachyons, no radiative electroweak symmetry breaking and, as $m_{0}$ gets larger, a Higgs potential that is unbounded from below.

Fig. [6] shows the 1D posterior PDFs for (a) $m_{0}$, (b) $m_{3 / 2}$ and (c) $\tan \beta$ for all three priors used with red (solid line) representing the linear priors, green (dashed lines) the log priors and blue (dotted lines) the natural priors. The dark matter constraint is asymmetric in all cases. As expected the logarithmic priors show a distinct preference for low $m_{0}$, with the linear priors expressing the same behaviour except for a longer and less suppressed tail to high $m_{0}$. The natural priors have the opposite behaviour, indicating that the fits are strongly prior dominated. Despite the aforementioned statistical noise, profile likelihoods do indicate that the region of low $m_{0}$ favoured by the linear and log priors is a better fit to the data than the region favoured by the natural priors. Indeed, part of this peak is likely due to a volume effect due to the large probability mass at high $m_{0}$. There is much better agreement between the three sets of priors for the posterior PDF for $m_{3 / 2}$ with all priors exhibiting a peak in the likelihood at near $50 \mathrm{TeV}$ falling off quite rapidly in the high $m_{3 / 2}$ region. The posterior $\mathrm{PDF}$ for $\tan \beta$ shows two different peaks. One of these is due to the natural priors and is at low $\tan \beta \sim 8$ due to the natural priors preference for low values of this variable. The other, broader peak is a better fit and occurs around $\tan \beta \sim 22$. This moderate value of $\tan \beta$ is preferred due to the statistical pull from observables such as $(g-2)_{\mu}, B R\left(B \rightarrow X_{s} \gamma\right)$ and $B R\left(B_{s} \rightarrow \mu^{+} \mu^{-}\right)$, and values higher than this are disfavoured due to the SM constraints on these same observables as is discussed in more detail in [26].

To summarize, like mGMSB, all of the mAMSB parameters show significant prior dependence, even when each is marginalized down to one dimension. We decline to present posterior PDFs of sparticle masses, since they show the same non-robustness with respect to changing the priors. We turn now to a more detailed examination of the constraints from the observables. 


\section{Constraining Power and Statistical Pull of Observ- ables}

\subsection{Information Content and Constraining Power}

In order to calculate the information gain in moving from a prior distribution to the posterior distribution, one can calculate the Kullback-Leibler (KL) [85] divergence (also called information divergence, information gain, or relative entropy) between the prior and the posterior distributions. Denoting the posterior distribution and prior distributions by $p(\underline{m} \mid \underline{d}, H)$ and $p(\underline{m} \mid H)$ respectively, the KL divergence of observable $i$ is defined as

$$
D_{K L}=\int p(\underline{m} \mid \underline{d}, H) \log \left(\frac{p(\underline{m} \mid \underline{d}, H)}{p(\underline{m})}\right) .
$$

$D_{K L}$ quantifies how much the prior PDF has been updated in its transformation to the posterior PDF. Using Eqs. 18,1, the KL divergence of observable $i$ is

$$
D_{\mathrm{KL}_{i}}=\int \frac{\mathcal{L}_{i}(\underline{m}) p(\underline{m} \mid H)}{p\left(d_{i} \mid H\right)} \log \left[\frac{\mathcal{L}_{i}(\underline{m})}{p\left(d_{i} \mid H\right)}\right] d \underline{m},
$$

where $p\left(d_{i} \mid H\right)=\int \mathcal{L}_{i}(\underline{m}) p(\underline{m} \mid H) d \underline{m}$. Since the Standard Model observables are used in our fits as inputs, we consider them to be a part of the prior. The KL divergence of all of the data combined $D_{\mathrm{KL}}$ is easily obtained from the MULTINEST algorithm which already calculates it to obtain the uncertainty on the evidence estimate (see [38]). We defined the constraining power of observable $i$ to be

$$
C_{P_{i}}=\frac{D_{\mathrm{KL}}}{D_{\mathrm{KL}}}
$$

The KL divergence was also employed in [10] to calculate the constraining power of observables for the $\mu>0$ branch of the CMSSM using linear and log priors and it was concluded that the information gain is dominated by the DM constraint which alone accounts for $C_{P}=0.8-0.95$ depending on the prior. By examining the variation of the $95 \% \mathrm{CL}$ regions in the CMSSM parameter space with and without the DM constraint, [18] on the other hand concluded that the DM has little role in constraining the parameter space of the CMSSM. We update the analysis of [10] by including the additional electroweak observables returned by SusyPOPE and calculating $D_{\mathrm{KL}}$ for the observables for the CMSSM with both signs of $\mu$. We list the $C_{P_{i}}$ values in Table 7 for the CMSSM, mAMSB and LVS respectively.

Table 7 shows that in the CMSSM, the DM constraint is dominant in constraining the parameter space, as is familiar from current literature. Since the asymmetric $L_{D M}$ rules out less

of parameter space than the symmetric constraint, $C_{P_{\Omega_{D M} h^{2}}}$ is always smaller in the asymmetric case. However, $B$-physics constraints dominated by $B R\left(B \rightarrow X_{s} \gamma\right)$, electroweak constraints, the anomalous magnetic moment of the muon and the Higgs mass constraint all play significant roles too. By contrast, mAMSB, which predicts approximately zero DM relic density over its entire parameter space, is hardly constrained at all by DM constraints. In fact, none of the indirect constraints provide much constraining power except for the electroweak observables. Once one has taken the SM inputs into account, the indirect observables 


\begin{tabular}{|c|c|c|c|c|c|c|}
\hline$C_{P_{i}}$ & \multicolumn{2}{|c|}{ symmetric $\mathcal{L}_{\text {DM }}$} & \multicolumn{3}{c|}{ asymmetric $\mathcal{L}_{\text {DM }}$} \\
\hline Observables/Prior & linear & log & natural & linear & log & natural \\
\hline \hline & \multicolumn{7}{|c|}{ CMSSM } \\
\hline$\Omega_{\text {DM }} h^{2}$ & 0.65 & 0.57 & 0.63 & 0.56 & 0.47 & 0.56 \\
B-Physics & 0.44 & 0.40 & 0.41 & 0.49 & 0.45 & 0.48 \\
$B R\left(B \rightarrow X_{s} \gamma\right)$ & 0.43 & 0.40 & 0.40 & 0.48 & 0.44 & 0.47 \\
Electroweak & 0.49 & 0.36 & 0.45 & 0.53 & 0.38 & 0.55 \\
$\delta a_{\mu}$ & 0.41 & 0.32 & 0.37 & 0.44 & 0.34 & 0.48 \\
$m_{h}$ & 0.42 & 0.39 & 0.39 & 0.46 & 0.42 & 0.47 \\
\hline \hline & 0.02 & 0.01 & 0.01 & 0.01 & 0.00 & 0.00 \\
$\Omega_{\text {DM }} h^{2}$ & 0.03 & 0.06 & 0.03 & 0.04 & 0.07 & 0.03 \\
B-Physics & 0.02 & 0.05 & 0.01 & 0.03 & 0.05 & 0.02 \\
$\left.B R \rightarrow X_{s} \gamma\right)$ & 0.12 & 0.11 & 0.14 & 0.13 & 0.11 & 0.15 \\
Electroweak & 0.01 & 0.05 & 0.01 & 0.02 & 0.06 & 0.02 \\
$\delta a_{\mu}$ & 0.02 & 0.05 & 0.04 & 0.03 & 0.05 & 0.05 \\
$m_{h}$ & \multicolumn{6}{|c|}{ LVS } \\
\hline$\Omega_{\text {DM }} h^{2}$ & 0.49 & 0.45 & 0.47 & 0.26 & 0.16 & 0.23 \\
B-Physics & 0.19 & 0.23 & 0.18 & 0.25 & 0.27 & 0.22 \\
$B R\left(B \rightarrow X_{s} \gamma\right)$ & 0.19 & 0.22 & 0.17 & 0.24 & 0.27 & 0.23 \\
Electroweak & 0.14 & 0.11 & 0.13 & 0.15 & 0.11 & 0.14 \\
$\delta a_{\mu}$ & 0.09 & 0.05 & 0.08 & 0.12 & 0.06 & 0.11 \\
$m_{h}$ & 0.20 & 0.20 & 0.21 & 0.25 & 0.25 & 0.26 \\
\hline \hline
\end{tabular}

Table 7: Constraining power of different observables in the CMSSM, mAMSB and LVS marginalized over both signs of $\mu$. 'B-Physics' includes the observables in the third section of Table 3. Electroweak observables include the ones in the second section of Table 3 plus $m_{W}$ and $\sin ^{2} \theta_{\text {eff }}^{l}$. Symmetric $\mathcal{L}_{\mathrm{DM}}$ denotes the assumption that the DM relic density is composed entirely of the LSP and asymmetric $\mathcal{L}_{\mathrm{DM}}$ codifies the assumption that the LSP forms only a part of the DM relic density. 
add very little to the degree of constraint on parameter space. The LVS is most highly constrained by DM, although less so than the CMSSM. This is because [13] a larger volume of the prior corresponds to a relic density compatible with the WMAP DM constraint compared to the CMSSM. All of the other indirect observables also help to constrain the LVS scenario in a non-trivial way. Defining $C_{S M}$ where $S M=\left\{\alpha_{s}\left(M_{Z}\right)^{\overline{M S}}, \alpha\left(M_{Z}\right)^{\overline{M S}}, m_{t}, m_{b}, m_{Z}\right\}$, we consistently find values $C_{S M}=0.8-0.9$ for each of the three models investigated in Table 7 , regardless of the form of the DM constraint or prior. It is therefore essential to vary these input parameters and to use the available experimental data to constrain them.

\subsection{Statistical Pull of Observables}

\subsection{1 mGMSB}

We now examine the pull exerted by the experimental constraints. Some of the more important of these are shown in Fig. 7. Considering first the posterior PDF for $m_{t}$, there is rough equality between the experimental constraint and that predicted by mGMSB, indicating that it is not being strongly pulled by other observables.

The $\mathrm{W}$ boson mass is typically under predicted by about $1 \sigma$ as compared to current direct measurements of its mass. The fits show a weak correlation between $m_{t}$ and $m_{W}$ such that a decrease in the experimental value of $m_{W}$ would also lead to a better fit for $m_{t}$. This effect is quite small however, as is the overall disagreement between theory and experiment. The PDFs for $m_{b}$ closely follows its experimental constraint and $\sin ^{2} \theta_{\text {eff }}^{l}$ is constrained to be close to its central value. These two observables are not shown, for brevity. In Fig. 7(c) we show the logarithm of the branching ratio for the flavour changing decay $B_{s} \rightarrow \mu^{+} \mu^{-}$. The $95 \%$ CL experimental upper limit from the Tevatron is marked with a black arrow at the right of the plot. If this branching ratio is measured to be non-zero in the near future, it will be far above the SM prediction and therefore provide a strong constraint on mGMSB, removing most of the currently available posterior density, which resides near the SM prediction of $\sim 10^{-8.5}$.

The $3.2 \sigma$ discrepancy between observation and the Standard Model theoretical value of the anomalous magnetic moment of the muon has received much attention from SUSY phenomenologists. In mGMSB the sign of the SUSY corrections to this quantity are correlated with the sign of $\mu$. The $\mu>0$ branch thus fits the data much better than the $\mu<0$ branch. For $\mu>0$ the natural priors prefer small values of $\delta a_{\mu}$, since the SUSY contributions to $\delta a_{\mu}$ are proportional to $\tan \beta$, large values of which are suppressed by the natural prior. $\log$ priors, while still exhibiting a preference for low values of $\delta a_{\mu}$, can provide a better fit.

Fig. [7(e) shows that mGMSB predicts the most likely branching ratio of $B \rightarrow X_{s} \gamma$ to be between $1 \sigma$ less than the experimental central value. The stop-chargino contribution interferes (for positive values of $M_{3}$ and $A_{t}$ such as we have in mGMSB) opposite to the sign of $\mu$ with respect to the SM contribution. When $\mu>0$ as preferred by $\delta a_{\mu}$, it destructively interferes. This is mitigated somewhat by the charged Higgs-top contribution, which always interferes constructively with the SM matrix element. The interplay between these two dominant SUSY contributions still leads to a predicted value below the observed one in 



Figure 7: The statistical pull of some important constraints on mGMSB, where no dark matter constraint is applied. Posterior PDFs are plotted for: red (solid) lines have linear priors, green (dashed) lines have log priors, blue (dotted) lines have natural priors and cyan (dash-dotted) is the experimental likelihood constraint. The figures show (a) the top mass $m_{t}$, (b) the $\mathrm{W}$ mass $m_{W}$, (c) the logarithm of the branching ratio for $B_{s} \rightarrow \mu^{+} \mu^{-}$with the black arrow being the current 95\% CL experimental upper limit, (d) the anomalous magnetic moment $\delta a_{\mu}$, (e) $B R\left(B \rightarrow X_{s} \gamma\right)$, (f) the branching ratio $B R\left(B_{u} \rightarrow \tau \nu\right)$ divided by its SM prediction and $(\mathrm{g})$ the isospin asymmetry in $B \rightarrow K^{*} \gamma$ decays $\Delta_{0-}$. 
mGMSB. For natural priors the PDF is quite sharply peaked around the SM value of $3.15 \times 10^{-4}$. For log and linear priors the peak is shifted slightly to lower values, with the tails of the distributions being longer than that of the natural priors.

Fig. $7(\mathrm{f})$ shows the posterior PDF of the ratio $R_{B \tau \nu} \equiv B R\left(B_{u} \rightarrow \tau \nu\right) / B R\left(B_{u} \rightarrow \tau \nu\right)_{S M}$, where $B R\left(B_{u} \rightarrow \tau \nu\right)_{S M}$ is the SM prediction of the branching ratio. The expressions for the SUSY corrections to this quantity can be found in [61, 62]. The SUSY contribution is always negative, so that the total MSSM prediction is smaller than that predicted by the Standard Model. The experimental value of $R_{B \tau \nu}^{e x p}=1.28 \pm 0.40$ is compatible with a non-zero SUSY contribution. However, a more precise experimental measurement of this value which had the same central value but half the uncertainty would be better fit without SUSY, and could lead to some tension with the $(g-2)_{\mu}$ measurement. The isospin asymmetry in $B \rightarrow K^{*} \gamma$ decays $\Delta_{0-}$ is shown in Fig. 7(g). mGMSB prefers a larger asymmetry than is observed. The discrepancy between the mode value and the experimental central value is just under $2 \sigma$.

We have also investigated the PDFs for the electroweak observables calculated using SusyPope. We have found that they do not exhibit enough variation over the parameter

space to present large constraints on our fits. In particular $R_{l}^{0}, R_{c}^{0}, R_{b}^{0}, \mathcal{A}_{b}, \mathcal{A}_{c}$ and $A_{\text {fb }}^{0, c}$ are all consistent with experiment independently of the prior. The forward backward asymmetry $A_{f b}^{0, b}$ is predicted too large by $2.4 \sigma$ while the left-right asymmetry $A_{L R}^{0}$ is too small by $2 \sigma$.

To summarize the mGMSB parameter space fits, we observe a similar significant level of prior dependence in mGMSB to that previously observed in the CMSSM [3, 4, 17, 6, 16, 10, 11]. It is clear from Figs. 3 and 4 that none of the parameters are constrained independently of prior and so the fits require additional more precise and/or direct data before they can be considered robust.

\subsection{2 mAMSB}

Fig. 8 shows the 1D posterior PDFs for some of the observables which constrain the mAMSB likelihood for the asymmetric $\mathcal{L}_{D M}$. A comparison with Fig. 7 shows that many of the posteriors of the observables are similar in the mAMSB and mGMSB cases. Only $\delta a_{\mu}$ and $B R\left(B \rightarrow X_{s} \gamma\right)$ show an large difference. The posterior PDF for $m_{t}$ in Fig. 8 (a) shows that, similarly to mGMSB, mAMSB is also in approximate agreement with the experimental constraint. Fig. 8(b) shows the posterior PDF for $m_{W}$ which is under-predicted by mAMSB similar to the other models we have considered. Fig. 8(c) shows the logarithm of the branching ratio for the process $B_{s} \rightarrow \mu^{+} \mu^{-}$. The central SM prediction lies at the peak of each posterior. Our prediction neglects flavour mixing in the squark sector which result from the AMSB soft-breaking terms [26]. Ref. [26] has shown that the effects of including squark flavour mixing can decrease $B R\left(B_{s} \rightarrow \mu^{+} \mu^{-}\right)$by up to a factor of two for $\tan \beta>22$. This would result in a shift of $\log _{10}\left(B R\left(B_{s} \rightarrow \mu^{+} \mu^{-}\right)\right.$in the plot by -0.3 . The $95 \%$ upper experimental limit placed on this process is $<5.8 \times 10^{-8}$ [65], which is always comfortably evaded even without this additional negative correction.

The SUSY contribution to the anomalous magnetic moment of the muon is presented in 

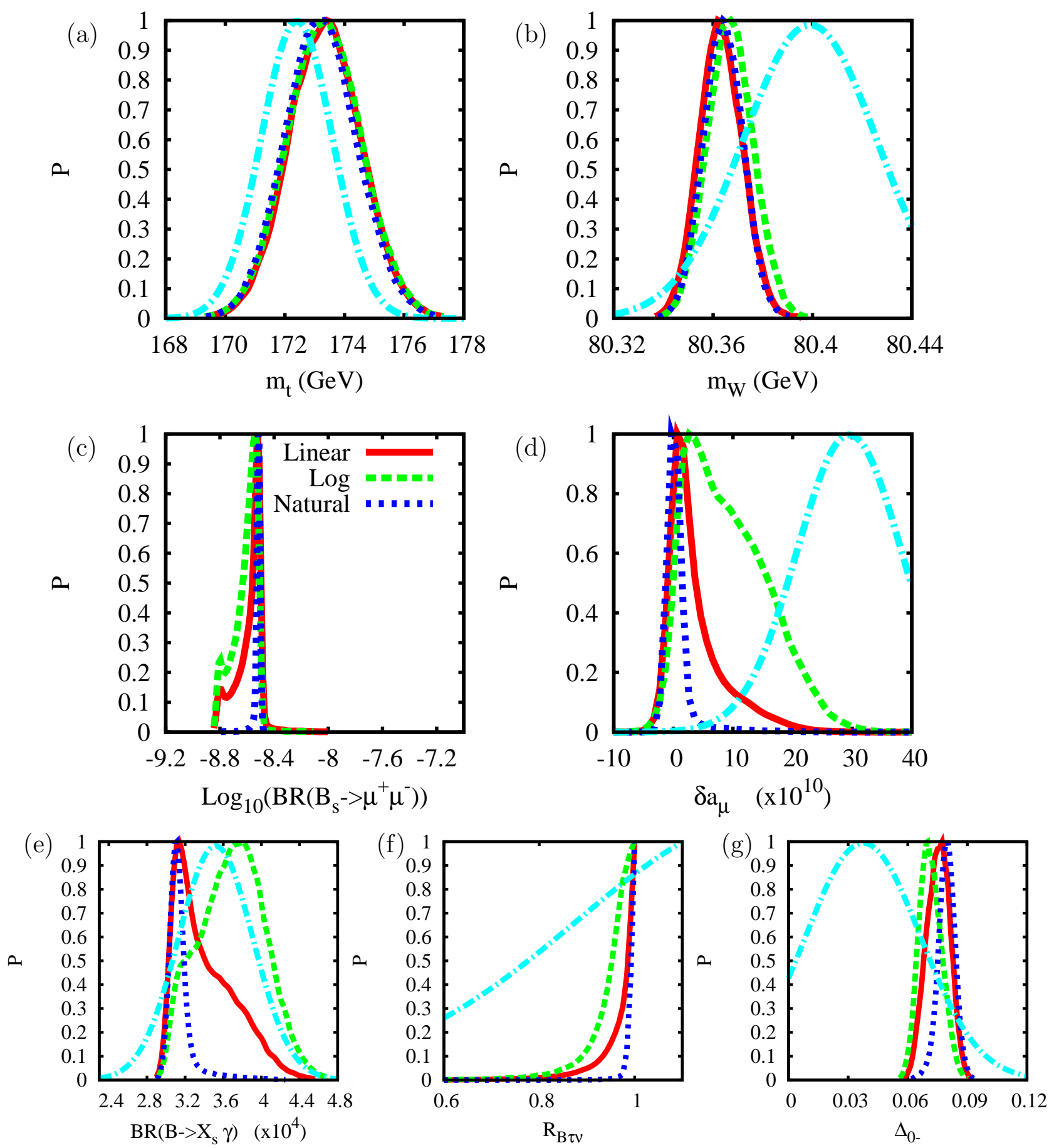

Figure 8: The statistical pull of some important constraints on mAMSB when the asymmetric dark matter constraint is applied. Posterior PDFs are plotted for: red (solid) lines have linear priors, green (dashed) lines have log priors, blue (dotted) lines have natural priors and cyan (dash-dotted) is the experimental likelihood constraint. The figures show (a) the top mass $m_{t}$, (b) the $\mathrm{W}$ mass $m_{W}$, (c) the logarithm of the branching ratio for $B_{s} \rightarrow \mu^{+} \mu^{-}$with the black arrow being the current 95\% CL experimental upper limit, (d) the anomalous magnetic moment $\delta a_{\mu}$, (e) $B R\left(B \rightarrow X_{s} \gamma\right)$, (f) the branching ratio $B R\left(B_{u} \rightarrow \tau \nu\right)$ divided by its SM prediction and $(\mathrm{g})$ the isospin asymmetry in $B \rightarrow K^{*} \gamma$ decays $\Delta_{0-}$. 
Fig. 8 (d). With natural priors, the SUSY contribution to the anomalous magnetic moment is very small due to heavy sparticles and low value of $\tan \beta$. Light sparticles and intermediate values of $\tan \beta$ favoured by the $\log$ priors allow a better fit to experiment. Larger values of $\tan \beta$, while being consistent with $\delta a_{\mu}$, would then lead to a worse fit to $B R\left(B \rightarrow X_{s} \gamma\right)$.

Squark flavour mixing corrections, which we neglected, can in principle have an effect on the $B R\left(B \rightarrow X_{s} \gamma\right)$ prediction in mAMSB, but the effect is small [26]. The $B R\left(B \rightarrow X_{s} \gamma\right)$ posterior PDF shown in Fig. 8(e) approximately follows the experimental constraint for log priors, in contrast to mGMSB. The natural priors case follows the experimental constraint less closely and the linear priors somewhere in between the natural and log prior cases. This behaviour is consistent with the values of the evidence in Table 4 , where the log priors allow a better fit than the other two priors.

The two observables shown in Fig. $8(\mathrm{f}, \mathrm{g})$ are the ratio $R_{B \tau \nu}$ and the isospin asymmetry in $B$ meson decays $\Delta_{0-}$, respectively. Both of these observables show the same behaviour as in mGMSB: not very constraining, due to the weakness of the large uncertainty in the SM prediction. While there is a small disagreement between the mAMSB prediction of $\Delta_{0-}$ and the experimental constraint there is too little variation of this observable over parameter space for it to mould the posterior significantly. The behaviour of the electroweak observables is qualitatively the same as in mGMSB, with all parameters except $A_{f b}^{0, b}$ and $A_{L R}^{0}$ being in good agreement with the data while showing little variation over parameter space. As the accuracy with which these parameters are known will not improve in the near future, we are skeptical that they will prove useful in constraining the parameter space of simple models such as mAMSB or mGMSB.

\subsection{Combined $\delta a_{\mu}-B R\left(B \rightarrow X_{s} \gamma\right)$ constraint}

It is interesting that mAMSB is the only model we study for which the SUSY contributions to $(g-2)_{\mu}$ and $B R\left(B \rightarrow X_{s} \gamma\right)$ are both probably of the right sign and magnitude to satisfy the experimental constraints simultaneously. There are a number of factors which contribute to this.

While the experimental and SM predictions for $B R\left(B \rightarrow X_{s} \gamma\right)$ are consistent with each other, the fact that the experimental result is larger than that predicted by the SM means that given a SUSY contribution to this observable of a specific magnitude, a positive contribution will always agree with the data better than a negative one. mAMSB with $\mu>0$ can accommodate such a contribution due to the sign of the coupling $A_{t}$. In mGMSB, mSUGRA and the LVS this will not be the case in the majority of parameter space. However, we note that using a more recent evaluation [86] of the Standard Model prediction which gives the branching ratio as $3.28 \pm 0.25 \times 10^{-4}$ would lessen this effect somewhat.

A further effect is the hierarchies present in mAMSB spectra compared to the relatively compressed mGMSB spectra. Recall from Eq. 14 that the magnitude of the supersymmetric one-loop $g-2$ correction scales as $\sim M_{1,2} \mu \tan \beta / m^{4}$, where $m$ is the mass scale of the relevant particles in the loop, i.e. the chargino/sneutrino or neutralino/smuon mass scale. The dominant SUSY contributions to $B R\left(B \rightarrow X_{s} \gamma\right)$ come from top-charged Higgs and 

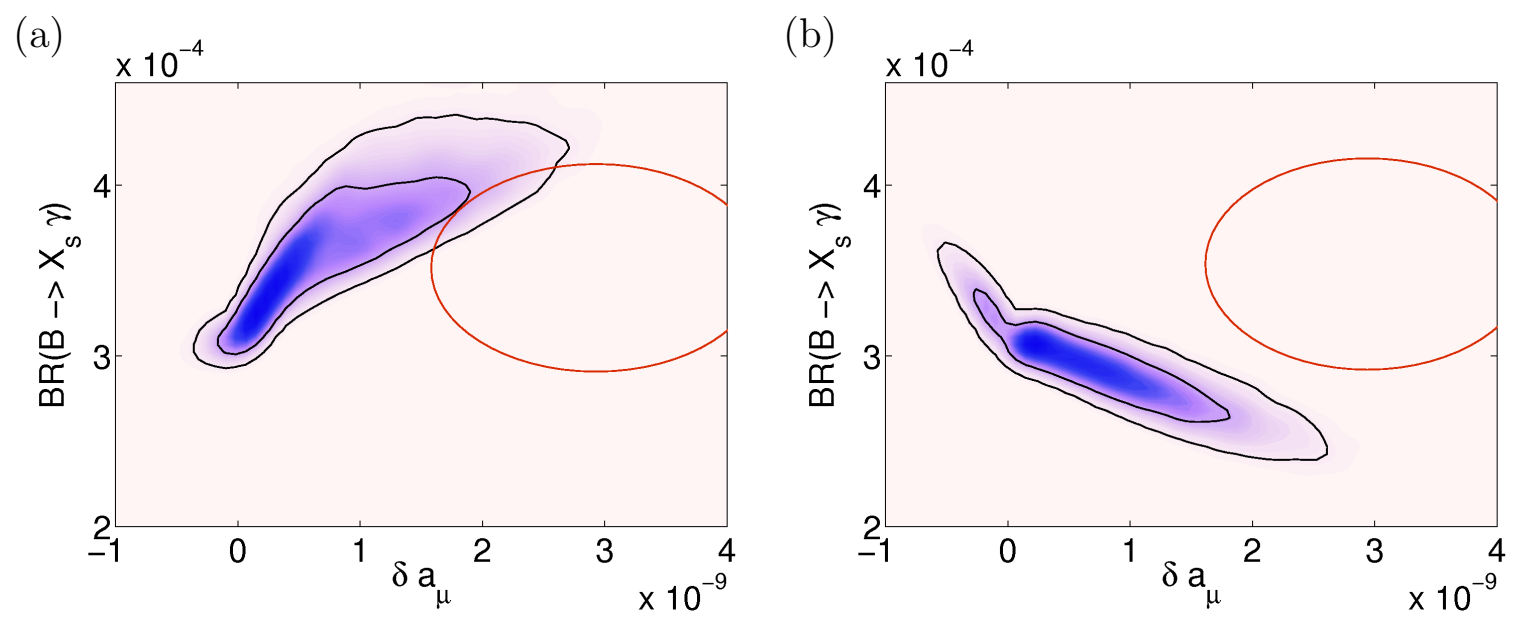

Figure 9: The 2D joint posterior in the $(g-2)_{\mu}-B R\left(B \rightarrow X_{s} \gamma\right)$ plane for (a) mAMSB and (b) mGMSB. Log priors and no dark matter constraint apply in both cases. $68 \%$ and $95 \%$ Bayesian credibility regions are shown as the inner and outer curves, respectively. The ellipse is centered on the experimental values of the two observables and show the $68 \%$ confidence level bounds on $\delta a_{\mu}$ and $B R\left(B \rightarrow X_{s} \gamma\right)$.

stop-chargino contributions. Agreement with the data typically prefers that the SUSY contribution to the branching ratio is not too large, i.e. the charged Higgs and stop masses are not too small. Thus, models in which the ratio of these masses to the slepton/neutralino masses is large have the potential to fit both $(g-2)_{\mu}$ and $B R\left(B \rightarrow X_{s} \gamma\right)$ simultaneously. In mGMSB, the spectrum is rather compressed compared to mAMSB, and so models with light neutralinos and sleptons also tend to have relatively light charged Higgs and stops. Thus, mGMSB fits the combination of the two observables less well than mAMSB. The interplay between $(g-2)_{\mu}$ and $B R\left(B \rightarrow X_{s} \gamma\right)$ fits has recently been explored in detail in [11] for the case of the CMSSM, and previously also in [87, 88].

We illustrate this behaviour in Fig. 9 which shows the 2D posterior PDFs in the $(g-$ $2)_{\mu^{-}} B R\left(B \rightarrow X_{s} \gamma\right)$ plane for mAMSB and mGMSB with log priors and no dark matter constraint. It shows the correlation between the two observables for mAMSB and anticorrelation for mGMSB, along with red ellipses showing the combined experimental constraint. This (anti-)correlation is expected from the signs of $A_{t}$ and $M_{3}$ in the models [26]. Parts of mAMSB parameter space that provide a good fit to all of the data are also within the error ellipse, contrary to the mGMSB case.

\subsection{Best-fit points}

Fig. 10 shows the best-fit points (defined to be the highest likelihood point) for mAMSB, mGMSB, the CMSSM and LVS. The asymmetric DM constraint was taken, except for mGMSB where no DM constraint was applied. The caption contains the parameters of each model that yielded the best-fit, and the bars on the right-hand side measure how far each observable is from its experimental central value. We should note that our fits contain 




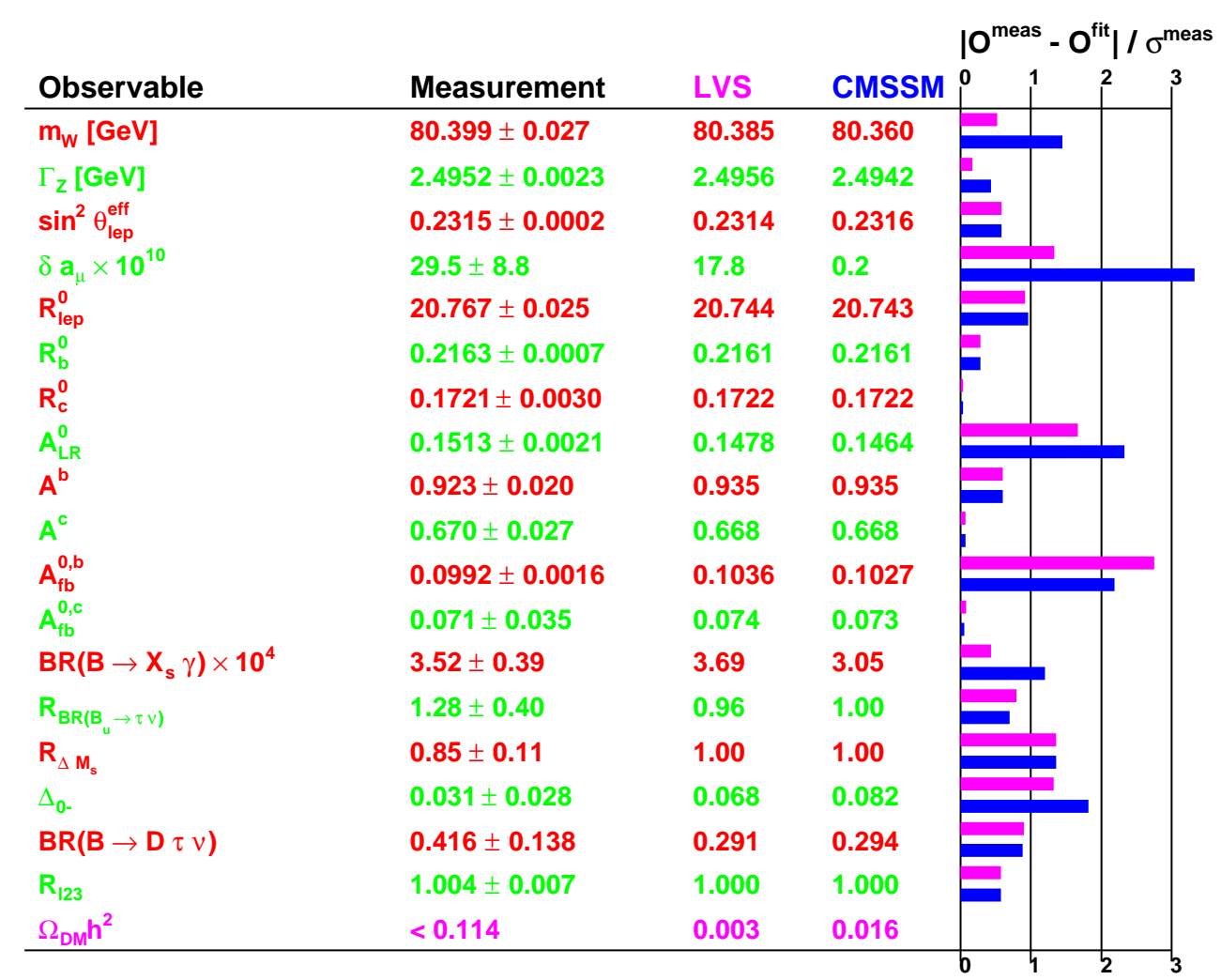

Figure 10: Best fit points. The best-fit point prediction and experimentally measured values for each observable are listed. The pulls, represented by the horizontal bars, are in units of standard deviations. The corresponding parameter points are: $m_{0}=312.7 \mathrm{GeV}, m_{3 / 2}=45$ $\mathrm{TeV}, \tan \beta=15.9$ for mAMSB, $N_{\text {mess }}=7, M_{\text {mess }}=1.23 \times 10^{7} \mathrm{GeV}, \Lambda=19.5 \mathrm{TeV} \tan \beta=$ 15.9 for mGMSB, $m_{1 / 2}=328 \mathrm{GeV}, \tan \beta=11.6$ for LVS and $m_{0}=3338 \mathrm{GeV}, m_{1 / 2}=382$ $\mathrm{GeV}, A_{0}=635, \tan \beta=8.6$ for the CMSSM. $\mu$ is positive for all of the best-fit points. 
quasi-degenerate likelihood maxima, and this combined with the fact that the MultiNesT algorithm is not optimized to find the best-fit point, may mean that the parameters of the best-fit point are poorly determined. This is not important for any of the Bayesian inferences we have presented, but it is instructive to examine the properties of some point in each model. The point sampled with the highest likelihood is a good candidate for the point to choose. The CMSSM best-fit point is in the focus point region [84]. It is not a really good fit to the anomalous magnetic moment of the muon, since heavy sleptons and moderate $\tan \beta$ render the SUSY contribution small. The other models' best-fit points all fit $\delta a_{\mu}$ much better, owing to their lighter spectra. Comparing the pulls of the electroweak observables, we see that there is not much variation in the $\chi^{2}$ values coming from them across the models except for $M_{W}$. The forward-backward asymmetry of $e^{+} e^{-} \rightarrow b \bar{b}, A_{f b}^{0, b}$, shows a small change, being a little larger in the LVS than the other models. The pull of the left-right asymmetry $A_{L R}^{0}$ also shows some small dependence. All of the models listed have a predicted relic density of dark matter much smaller than the WMAP-inferred central value, requiring a dominant component of non neutralino dark matter (except for mGMSB, where we do not apply the DM constraint).

\section{Conclusion}

We ask the question: are any of the most commonly assumed low-parameter SUSY breaking mediation mechanisms favored over the others by current indirect and cosmological data? Aside from direct searches, SUSY corrections contribute to the observables we consider only in loop effects. For observables that agree with the standard model prediction, arbitrarily heavy sparticles suppress such loop effects and so will fit that observable. However, the anomalous magnetic moment of the muon prefers a significant contribution from supersymmetric loops, and so this observable prefers light SUSY particles. The dark matter constraint from cosmological observations cannot be accounted for by the Standard Model, but can be fitted by the MSSM if the lightest supersymmetric particle is the neutralino. We argue that the Bayesian evidence is the relevant statistical quantity for such an analysis.

When performing such a statistical global fit, it is important to check robustness. The fits should be dominated by the data and not by the form of the prior if we are to claim robustness. Previous studies showed that fits to the CMSSM with four extra parameters are prior dependent [4, 6, 10, whereas the LVS model [13] (with only two extra parameters) is more robust. Thus it was natural to select mGMSB and mAMSB, each of which have three extra parameters to check robustness of the fits and compare the models against each other. One expects the level of robustness to go down with higher numbers of parameters, which a recent fit to the phenomenological MSSM [14] with twenty extra parameters, illustrated]. We have presented constraints on the mGMSB and mAMSB parameter spaces, and found that in both cases there is significant prior dependence. Parameter inference from the models are therefore not robust and therefore require further more precise and direct data, perhaps from collider measurements of SUSY particles. Unsurprisingly, no robust statement regarding the

\footnotetext{
${ }^{4}$ There were a couple of prior independent inferences in the fits, such as the lightest CP-even Higgs mass.
} 
sign of $\mu$ can be made for mAMSB or mGMSB. We therefore encourage that future work on mAMSB and mGMSB include both signs of $\mu$ until the data is strong enough to support a prior-independent pronouncement on the status of the sign of $\mu$, and not to disregard the $\mu<0$ branch of the theory based on the preference of one observable for $\mu>0$. The Large Volume Scenario, with two fewer parameters than the CMSSM is more constrained and shows a robust moderate preference for $\mu>0$.

The model preferred by the data depends on what we assume for the DM relic density: whether it is made entirely of neutralinos (symmetric constraint) or whether we allow for the presence of non-neutralino dark matter (asymmetric constraint). An analysis of the constraining power of the various observables showed that it resides dominantly in the DM constraint in the case of the CMSSM and the LVS. This is not the case in mAMSB where the relic density is uniformly too small by an order of magnitude across parameter space, and the main constraint comes from the combined electroweak observables. Dropping the DM constraint altogether allows a comparison with mGMSB, but then no strong robust preference for any model can be found. However, for the symmetric constraint, mAMSB is strongly disfavoured (since it predicts essentially no neutralino dark matter) over the CMSSM and LVS. With the asymmetric constraint, mAMSB is at least moderately favoured over the CMSSM.

Experience and familiarity with the methods of model selection and Bayesian inference from work such as that contained here will be invaluable once further more constraining data become available, hopefully from SUSY signals at colliders.

\section{Acknowledgements}

MJD wishes to thank St. John's College, the CET and EPSRC for support. SSA is supported by the Gates Cambridge Trust, and thanks F. Quevedo and D. J. C. McKay for discussions. We thank A. Casas and C. Lester for discussions about the MSSM prior and P. Slavich for communication about $B R\left(B \rightarrow X_{s} \gamma\right)$. This work was performed using the Darwin Supercomputer of the University of Cambridge High Performance Computing Service (http://www.hpc.cam.ac.uk/), provided by Dell Inc. using Strategic Research Infrastructure Funding from the Higher Education Funding Council for England. The original idea for this project came from discussions with S. Kraml and others in the TeV Colliders Les Houches workshop in 2007. This work was partially supported by STFC.

\section{A Dark Matter Direct Detection}

In this appendix we present results on DM direct detection cross-sections for the Large Volume Scenario. Direct detection rates in the CMSSM have been most recently been presented in Bayesian global fits in Refs. [8, 9]. Our updated CMSSM fits are similar to the results of those articles, so we do not include them here. Our calculations of the detection cross-section have been obtained with micrOMEGAS2.3.1[47, 48, 49, 50]. 
(a)

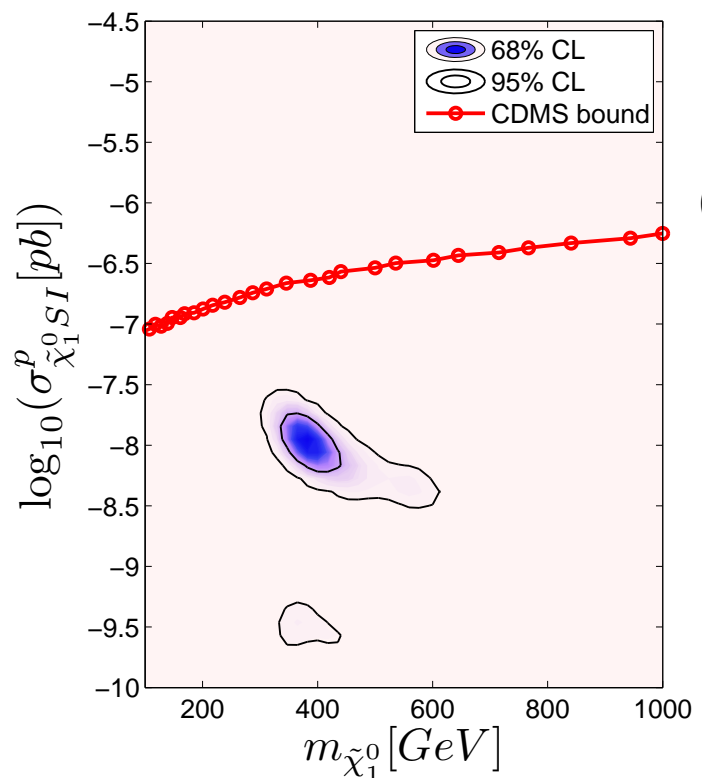

(b)

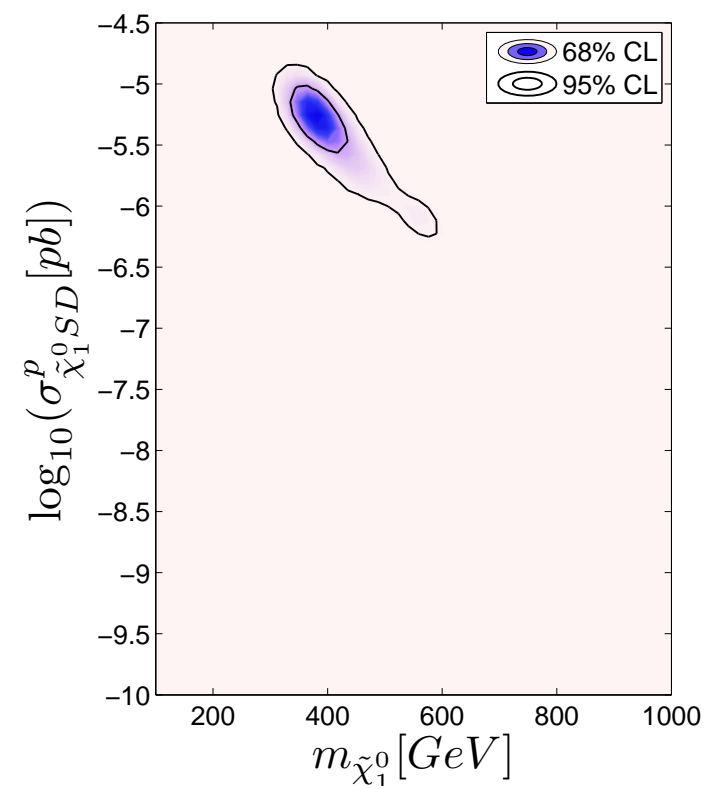

Figure 11: Posterior PDFs with linear priors for (a) the spin-independent cross-section $\sigma_{S I}$ and (b) the spin-dependent cross-section $\sigma_{S D}$ for neutralino-nucleon elastic scattering plotted against the neutralino mass $m_{\chi}$ in the Large Volume Scenario, marginalized over both signs of $\mu$. Both plots have a symmetric dark matter constraint.

Neutralinos are Majorana fermions and can therefore interact with quarks via scalar and axial-vector interactions, but not via a vector-like interaction. In the scalar (spinindependent) case, the neutralino can interact at tree-level with quarks in the nucleus via Higgs or squark exchange. At one-loop the neutralino can couple to gluons via a quark/squark loop. At zero momentum transfer, the total cross-section is given by

$$
\sigma=\frac{4 m_{r e d}^{2}}{\pi}\left(Z f_{p}+(A-Z) f_{n}\right)^{2}
$$

where $m_{r e d}$ is the nucleon-neutralino reduced mass, $Z$ and $(A-Z)$ are the number of protons and neutrons in the nucleus and $f_{p(n)}$ is the coupling of the neutralino to the proton(neutron) respectively.

The neutralino-quark axial-vector interaction leads to a spin-dependent coupling proportional to $J(J+1)$, where $J$ is the spin of the nucleus. The cross-section is given by

$$
\frac{d \sigma}{d|\vec{v}|^{2}}=\frac{1}{2 \pi v^{2}} \overline{\left|T\left(v^{2}\right)\right|^{2}}
$$

where $v$ is the relative velocity of the neutralino and $T\left(v^{2}\right)$ is the scattering matrix element. This expression is then integrated over the Boltzmann velocity distribution of the neutralinos to obtain an average cross section.

Since the results of our fits indicate that the LVS is sufficiently constrained so as to be independent of the prior, we show only the case of linear priors. The cross-sections are independent of whether the nucleon is a proton or neutron. We therefore have plotted the average $\left(\sigma^{p}+\sigma^{n}\right) / 2$ for each point sampled. As elsewhere in this article we marginalize over the sign of $\mu$.

Fig. 11(a) shows the 2D posterior PDF for the logarithm of the spin-independent crosssection per nucleon in picobarns against the neutralino mass $m_{\chi}$. We also show the CDMS [89] 
upper bound assuming the local DM density to be $\rho_{0}=0.3 \mathrm{Gev} / \mathrm{cm}^{3}$. Fig. 11(b) shows the posterior PDF for the log of the spin-dependent cross-section per nucleon versus the neutralino mass. The DM detection cross-sections are both well below the bounds set by the current generation of DM searches such as CDMS[89] in the spin-independent and SuperK [90] in the spin-dependent cases. Future one-tonne detectors should be able to probe the entire range of the LVS parameter space for the spin-independent cross-section down to $\sigma_{S I} \sim 10^{-10} \mathrm{pb}$. The spin-dependent result is independent of the sign of $\mu$ giving a robust prediction of $\sigma_{S D} \sim 10^{-5.5 \pm 0.5} \mathrm{pb}$.

\section{References}

[1] P. Fayet, Supersymmetry and Weak, Electromagnetic and Strong Interactions, Phys. Lett. B64 (1976) 159.

[2] S. Dimopoulos and H. Georgi, Softly Broken Supersymmetry and SU(5), Nucl. Phys. B193 (1981) 150.

[3] B. C. Allanach and C. G. Lester, Multi-Dimensional mSUGRA Likelihood Maps, Phys. Rev. D73 (2006) 015013, hep-ph/0507283.

[4] B. C. Allanach, Naturalness priors and fits to the constrained minimal supersymmetric standard model, Phys. Lett. B635 (2006) 123-130, hep-ph/0601089.

[5] R. R. de Austri, R. Trotta, and L. Roszkowski, A Markov chain Monte Carlo analysis of the CMSSM, JHEP 05 (2006) 002, hep-ph/0602028.

[6] B. C. Allanach, C. G. Lester, and A. M. Weber, The Dark Side of mSUGRA, JHEP 12 (2006) 065, hep-ph/0609295.

[7] B. C. Allanach, K. Cranmer, C. G. Lester, and A. M. Weber, Natural Priors, CMSSM Fits and LHC Weather Forecasts, JHEP 08 (2007) 023, [arXiv:0705.0487].

[8] L. Roszkowski, R. Ruiz de Austri, and R. Trotta, Implications for the Constrained $M S S M$ from a new prediction for $b \rightarrow s \gamma$, JHEP 07 (2007) 075, [arXiv:0705.2012].

[9] B. C. Allanach and D. Hooper, Panglossian Prospects for Detecting Neutralino Dark Matter in Light of Natural Priors, JHEP 10 (2008) 071, [arXiv:0806.1923].

[10] R. Trotta, F. Feroz, M. P. Hobson, L. Roszkowski, and R. Ruiz de Austri, The Impact of priors and observables on parameter inferences in the Constrained MSSM, JHEP 12 (2008) 024, [arXiv:0809.3792].

[11] F. Feroz, M. P. Hobson, L. Roszkowski, R. Ruiz de Austri, and R. Trotta, Are $B R(b \rightarrow s \gamma)$ and $(g-2)_{\mu}$ consistent within the Constrained MSSM?, arXiv:0903.2487. 
[12] R. Trotta, R. R. de Austri and C. P. d. Heros, Prospects for dark matter detection with IceCube in the context of the CMSSM, arXiv:0906.0366 [astro-ph.HE].

[13] B. C. Allanach, M. J. Dolan, and A. M. Weber, Global Fits of the Large Volume String Scenario to WMAP5 and Other Indirect Constraints Using Markov Chain Monte Carlo, JHEP 08 (2008) 105, [arXiv:0806.1184].

[14] S. S. AbdusSalam, B. C. Allanach, F. Quevedo, F. Feroz, and M. Hobson, Fitting the Phenomenological MSSM, [arXiv:0904.2548].

[15] L. Roszkowski, R. Ruiz de Austri, R. Trotta, Y.-L. S. Tsai, and T. Varley, Some novel features of the Non-Universal Higgs Model, [arXiv:0903.1279]

[16] F. Feroz et. al., Bayesian Selection of sign(mu) within mSUGRA in Global Fits Including WMAP5 Results, JHEP 10 (2008) 064, [arXiv:0807.4512].

[17] O. Buchmueller et. al., Prediction for the Lightest Higgs Boson Mass in the CMSSM using Indirect Experimental Constraints, Phys. Lett. B657 (2007) 87-94, [arXiv:0707.3447].

[18] O. Buchmueller et. al., Predictions for Supersymmetric Particle Masses in the CMSSM using Indirect Experimental and Cosmological Constraints, JHEP 09 (2008) 117, [arXiv:0808.4128].

[19] S. Heinemeyer, X. Miao, S. Su, and G. Weiglein, $B^{-}$Physics Observables and Electroweak Precision Data in the CMSSM, mGMSB and mAMSB, JHEP 08 (2008) 087, [arXiv:0805.2359].

[20] L. Randall and R. Sundrum, Out of this world supersymmetry breaking, Nucl. Phys. B557 (1999) 79-118, hep-th/9810155.

[21] G. F. Giudice and R. Rattazzi, Theories with gauge-mediated supersymmetry breaking, Phys. Rept. 322 (1999) 419-499, hep-ph/9801271.

[22] V. Balasubramanian, P. Berglund, J. P. Conlon, and F. Quevedo, Systematics of Moduli Stabilisation in Calabi-Yau Flux Compactifications, JHEP 03 (2005) 007, hep-th/0502058.

[23] J. P. Conlon, F. Quevedo, and K. Suruliz, Large-volume flux compactifications: Moduli spectrum and D3/D7 soft supersymmetry breaking, JHEP 08 (2005) 007, hep-th/0505076.

[24] J. P. Conlon, S. S. Abdussalam, F. Quevedo, and K. Suruliz, Soft SUSY breaking terms for chiral matter in IIB string compactifications, JHEP 01 (2007) 032, hep-th/0610129.

[25] S. S. AbdusSalam, J. P. Conlon, F. Quevedo, and K. Suruliz, Scanning the Landscape of Flux Compactifications: Vacuum Structure and Soft Supersymmetry Breaking, JHEP 12 (2007) 036, [arXiv:0709.0221]. 
[26] B. C. Allanach, G. Hiller, D. R. T. Jones, and P. Slavich, Flavour Violation in Anomaly Mediated Supersymmetry Breaking, arXiv:0902.4880.

[27] R. T. Cox Am. J. Phys. vol. 14, pp. 1-13, 1946.

[28] F. Feroz, M. P. Hobson, J. T. L. Zwart, R. D. E. Saunders, and K. J. B. Grainge, Bayesian modelling of clusters of galaxies from multi- frequency pointed Sunyaev-Zel'dovich observations, arXiv:0811.1199.

[29] F. Feroz, P. J. Marshall, and M. P. Hobson, Cluster detection in weak lensing surveys, arXiv:0810.0781.

[30] J. Ó Ruanaidh and W. Fitzgerald, Numerical Bayesian Methods Applied to Signal Processing. Springer Verlag:New York, 1996.

[31] M. P. Hobson, S. L. Bridle, and O. Lahav, Combining cosmological datasets: hyperparameters and Bayesian evidence, Mon. Not. Roy. Astron. Soc. 335 (2002) 377, astro-ph/0203259.

[32] R. M. Neal, Estimating ratios of normalizing constants using linked importance sampling, 2005.

[33] Muon G-2 Collaboration, G. W. Bennett et. al., Final report of the muon E821 anomalous magnetic moment measurement at BNL, Phys. Rev. D73 (2006) 072003, hep-ex/0602035.

[34] A. Gelman and X. Meng, Simulating normalizing constants: From importance sampling to bridge sampling to path sampling, 1998.

[35] B. C. Allanach and C. G. Lester, Sampling using a 'bank' of clues, Comput. Phys. Commun. 179 (2008) 256-266, [arXiv:0705.0486].

[36] A. R. Liddle, Information criteria for astrophysical model selection, Mon. Not. Roy. Astron. Soc. Lett. 377 (2007) L74-L78, astro-ph/0701113.

[37] J. Skilling, Nested Sampling, in American Institute of Physics Conference Series (R. Fischer, R. Preuss, and U. V. Toussaint, eds.), pp. 395-405, Nov., 2004.

[38] F. Feroz and M. P. Hobson, Multimodal nested sampling: an efficient and robust alternative to $M C M C$ methods for astronomical data analysis, arXiv:0704.3704.

[39] F. Feroz, M. P. Hobson, and M. Bridges, MultiNest: an efficient and robust Bayesian inference tool for cosmology and particle physics, arXiv:0809.3437.

[40] R. Trotta, Bayes in the sky: Bayesian inference and model selection in cosmology, Contemp. Phys. 49 (2008) 71-104, [arXiv:0803.4089].

[41] R. D. Cousins, Comment on 'Bayesian Analysis of Pentaquark Signals from CLAS Data', with Response to the Reply by Ireland and Protopopsecu, Phys. Rev. Lett. 101 (2008) 029101, [arXiv:0807.1330]. 
[42] M. E. Cabrera, J. A. Casas, and R. Ruiz de Austri, Bayesian approach and Naturalness in MSSM analyses for the LHC, JHEP 03 (2009) 075, [arXiv:0812.0536].

[43] S. Heinemeyer, W. Hollik, D. Stockinger, A. M. Weber, and G. Weiglein, Precise prediction for $M_{W}$ in the MSSM, JHEP 08 (2006) 052, hep-ph/0604147].

[44] S. Heinemeyer, W. Hollik, A. M. Weber, and G. Weiglein, Z Pole Observables in the MSSM, JHEP 04 (2008) 039, [arXiv:0710.2972].

[45] CDF Collaboration, Combination of CDF and D0 results on the $W$ boson mass and width, arXiv:0808.0147.

[46] LEP Collaboration, J. Alcaraz et. al., Precision Electroweak Measurements and Constraints on the Standard Model, arXiv:0712.0929.

[47] G. Belanger, F. Boudjema, A. Pukhov, and A. Semenov, Dark matter direct detection rate in a generic model with micrOMEGAs2.1, arXiv:0803.2360.

[48] G. Belanger, F. Boudjema, A. Pukhov, and A. Semenov, micrOMEGAs2.0: A program to calculate the relic density of dark matter in a generic model, Comput. Phys. Commun. 176 (2007) 367-382, hep-ph/0607059.

[49] G. Belanger, F. Boudjema, A. Pukhov, and A. Semenov, micrOMEGAs: Version 1.3, Comput. Phys. Commun. 174 (2006) 577-604, hep-ph/0405253.

[50] G. Belanger, F. Boudjema, A. Pukhov, and A. Semenov, micrOMEGAs: A program for calculating the relic density in the MSSM, Comput. Phys. Commun. 149 (2002) 103-120, hep-ph/0112278.

[51] S. Heinemeyer, D. Stockinger, and G. Weiglein, Electroweak and supersymmetric two-loop corrections to $(g-2)_{\mu}$, Nucl. Phys. B699 (2004) 103-123, hep-ph/0405255.

[52] S. Heinemeyer, D. Stockinger, and G. Weiglein, Two-loop SUSY corrections to the anomalous magnetic moment of the muon, Nucl. Phys. B690 (2004) 62-80, hep-ph/0312264.

[53] D. Stockinger, The muon magnetic moment and supersymmetry, J. Phys. G34 (2007) R45-R92, hep-ph/0609168.

[54] S. Marchetti, S. Mertens, U. Nierste, and D. Stockinger, Tan(beta)-enhanced supersymmetric corrections to the anomalous magnetic moment of the muon, Phys. Rev. D79 (2009) 013010, [arXiv:0808.1530].

[55] Particle Data Group Collaboration, C. Amsler et. al., Review of particle physics, Phys. Lett. B667 (2008) 1.

[56] WMAP Collaboration, E. Komatsu et. al., Five-Year Wilkinson Microwave Anisotropy Probe (WMAP5) Observations:Cosmological Interpretation, Astrophys. J. Suppl. 180 (2009) 330-376, [arXiv:0803.0547]. 
[57] B. C. Allanach, A. Djouadi, J. L. Kneur, W. Porod, and P. Slavich, Precise determination of the neutral Higgs boson masses in the MSSM, JHEP 09 (2004) 044, hep-ph/0406166.

[58] LEP Working Group for Higgs boson searches Collaboration, R. Barate et. al., Search for the standard model Higgs boson at LEP, Phys. Lett. B565 (2003) 61-75, hep-ex/0306033.

[59] ALEPH Collaboration et al., Precision electroweak measurements on the $Z$ resonance, Phys. Rept. 427 (2006) 257, hep-ex/0509008.

[60] Dmitri Yu. Bardin et al., Electroweak Working Group Report, hep-ph/9707229

[61] F. Mahmoudi, SuperIso: A program for calculating the isospin asymmetry of $B \rightarrow K^{*} \gamma$ in the MSSM, Comput. Phys. Commun. 178 (2008) 745-754, [arXiv:0710.2067].

[62] F. Mahmoudi, SuperIso v2.3: A Program for calculating flavor physics observables in Supersymmetry, arXiv:0808.3144.

[63] Heavy Flavor Averaging Group Collaboration, E. Barberio et. al., Averages of b-hadron and c-hadron Properties at the End of 2007, arXiv:0808.1297.

[64] We thank C S Lin for providing us with the likelihoods.

[65] CDF Collaboration, T. Aaltonen et. al., Search for $B_{s}^{0} \rightarrow \mu^{+} \mu^{-}$and $B_{d}^{0} \rightarrow \mu^{+} \mu^{-}$ decays with $2 \mathrm{fb}^{-1}$ of p p collisions, Phys. Rev. Lett. 100 (2008) 101802, [arXiv:0712.1708].

[66] BABAR Collaboration, B. Aubert et. al., Observation of the semileptonic decays $B \rightarrow D^{*} \tau^{-} \bar{\nu}_{\tau}$ and evidence for $B \rightarrow D \tau^{-} \bar{\nu}_{\tau}$, Phys. Rev. Lett. 100 (2008) 021801, [arXiv:0709.1698].

[67] G. Isidori and P. Paradisi, Hints of large tan(beta) in flavour physics, Phys. Lett. B639 (2006) 499-507, hep-ph/0605012].

[68] A. J. Buras, P. H. Chankowski, J. Rosiek, and L. Slawianowska, $\Delta M_{d, s}, B^{0} d, s \rightarrow \mu^{+} \mu^{-}$and $B \rightarrow X_{s} \gamma$ in supersymmetry at large $\tan \beta$, Nucl. Phys. B659 (2003) 3, hep-ph/0210145.

[69] UTfit Collaboration, M. Bona et. al., The unitarity triangle fit in the standard model and hadronic parameters from lattice QCD: A reappraisal after the measurements of $\operatorname{Delta}(m(s))$ and $B R\left(B \rightarrow \tau \nu_{\tau}\right)$, JHEP 10 (2006) 081, hep-ph/0606167].

[70] BABAR Collaboration, B. Aubert et. al., Measurement of Branching Fractions and $C P$ and Isospin Asymmetries in $B \rightarrow K^{*} \gamma$, arXiv:0808.1915.

[71] BELLE Collaboration, M. Nakao et. al., Measurement of the $B \rightarrow K^{*} \gamma$ branching fractions and asymmetries, Phys. Rev. D69 (2004) 112001, hep-ex/0402042. 
[72] FlaviaNet Working Group on Kaon Decays Collaboration, M. Antonelli et. al., Precision tests of the Standard Model with leptonic and semileptonic kaon decays, arXiv:0801.1817.

[73] Tevatron Electroweak Working Group Collaboration, Combination of CDF and D0 Results on the Mass of the Top Quark, arXiv:0808.1089.

[74] T. Becher and M. D. Schwartz, A Precise determination of $\alpha_{s}$ from LEP thrust data using effective field theory, JHEP 07 (2008) 034, [arXiv:0803.0342].

[75] J. P. Conlon and F. Quevedo, Astrophysical and Cosmological Implications of Large Volume String Compactifications, JCAP 0708 (2007) 019, [arXiv:0705.3460].

[76] N. Baro, F. Boudjema, and A. Semenov, Full one-loop corrections to the relic density in the MSSM: A few examples, Phys. Lett. B660 (2008) 550-560, [arXiv:0710.1821].

[77] H. Pagels and J. R. Primack, Supersymmetry, Cosmology and New TeV Physics, Phys. Rev. Lett. 48 (1982) 223.

[78] N. Yoshida, A. Sokasian, L. Hernquist, and V. Springel, Early Structure Formation and Reionization in a Warm Dark Matter Cosmology, Astrophys. J. 591 (2003) L1-L4, astro-ph/0303622.

[79] B. C. Allanach, SOFTSUSY: A $C++$ program for calculating supersymmetric spectra, Comput. Phys. Commun. 143 (2002) 305-331, hep-ph/0104145.

[80] T. Gherghetta, G. F. Giudice, and J. D. Wells, Phenomenological consequences of supersymmetry with anomaly-induced masses, Nucl. Phys. B559 (1999) 27-47, hep-ph/9904378.

[81] P. Skands et. al., SUSY Les Houches accord: Interfacing SUSY spectrum calculators, decay packages, and event generators, JHEP 07 (2004) 036, hep-ph/0311123.

[82] G. Degrassi, P. Gambino, and P. Slavich, SusyBSG: a fortran code for $B R\left[B \rightarrow X_{s} \gamma\right]$ in the MSSM with Minimal Flavor Violation, Comput. Phys. Commun. 179 (2008) 759-771, [arXiv:0712.3265].

[83] J. A. Bagger, K. T. Matchev, D. M. Pierce, and R.-j. Zhang, Weak-scale phenomenology in models with gauge-mediated supersymmetry breaking, Phys. Rev. D55 (1997) 3188-3200, hep-ph/9609444.

[84] J. L. Feng, K. T. Matchev, and T. Moroi, Focus points and naturalness in supersymmetry, Phys. Rev. D61 (2000) 075005, hep-ph/9909334.

[85] S. Kullback and R. A. Leibler (1951).On Information and Sufficiency, The Annals of Mathematical Statistics 22 (1951): 79

[86] P. Gambino and P. Giordano, Normalizing inclusive rare B decays, Phys. Lett. B669 (2008) 69, [0805.0271]. 
[87] H. Baer and others, Updated constraints on the minimal supergravity model, JHEP 0207 (2002) 050, hep-ph/0205325.

[88] H. Baer, A. Belyaev, T. Krupovnikas and M. Mustafayev, SUSY normal scalar mass hierarchy reconciles $(g-2)_{\mu}, b \rightarrow s \gamma$ and relic density JHEP 0406 (2004) 044, hep-ph/0403214.

[89] CDMS Collaboration, Z. Ahmed et. al., Search for Weakly Interacting Massive Particles with the First Five-Tower Data from the Cryogenic Dark Matter Search at the Soudan Underground Laboratory, Phys. Rev. Lett. 102 (2009) 011301, arXiv:0802.3530].

[90] Super-Kamiokande Collaboration, S. Desai et. al., Search for dark matter WIMPs using upward through-going muons in Super-Kamiokande, Phys. Rev. D70 (2004) 083523, hep-ex/0404025. 